\title{
Infinite-dimensional Log-Determinant divergences between positive definite Hilbert-Schmidt operators
}

\author{
Hà Quang Minh
}

Istituto Italiano di Tecnologia, Via Morego 30, Genova 16163, ITALY

\begin{abstract}
The current work generalizes the author's previous work on the infinite-dimensional Alpha Log-Determinant (Log-Det) divergences and Alpha-Beta Log-Det divergences, defined on the set of positive definite unitized trace class operators on a Hilbert space, to the entire Hilbert manifold of positive definite unitized Hilbert-Schmidt operators. This generalization is carried out via the introduction of the extended Hilbert-Carleman determinant for unitized Hilbert-Schmidt operators, in addition to the previously introduced extended Fredholm determinant for unitized trace class operators. The resulting parametrized family of Alpha-Beta Log-Det divergences is general and contains many divergences between positive definite unitized Hilbert-Schmidt operators as special cases, including the infinite-dimensional affine-invariant Riemannian distance and the infinite-dimensional generalization of the symmetric Stein divergence.
\end{abstract}

Keywords:

2010 MSC: 47B65, 47L07, 46E22, 15A15

infinite-dimensional Log-Determinant divergences, Alpha-Beta divergences, affine-invariant Riemannian distance, positive definite operators, Hilbert-Schmidt operators, extended Hilbert-Carleman determinant, trace class operators, extended Fredholm determinant

Email address: minh. haquang@iit. it (Hà Quang Minh) 


\section{Introduction}

The current work is a continuation and generalization of the author's previous work [1], [2], which generalizes the finite-dimensional Log-Determinant divergences to the infinite-dimensional setting. We recall that for the convex cone $\operatorname{Sym}^{++}(n)$ of symmetric, positive definite (SPD) matrices of size $n \times n, n \in \mathbb{N}$, the Alpha-Beta LogDeterminant (Log-Det) divergence between $A, B \in \mathrm{Sym}^{++}(n)$ is a parametrized family of divergences defined by (see [3])

$$
D^{(\alpha, \beta)}(A, B)=\frac{1}{\alpha \beta} \log \operatorname{det}\left[\frac{\alpha\left(A B^{-1}\right)^{\beta}+\beta\left(A B^{-1}\right)^{-\alpha}}{\alpha+\beta}\right], \alpha>0, \beta>0,
$$

along with the limiting cases $(\alpha>0, \beta=0),(\alpha=0, \beta>0)$, and $(\alpha=0, \beta=0)$. This family contains many distance-like functions on $\mathrm{Sym}^{++}(n)$, including

1. The affine-invariant Riemannian distance $d_{\mathrm{aiE}}[4]$, corresponding to

$$
D^{(0,0)}(A, B)=\frac{1}{2} d_{\mathrm{aiE}}^{2}(A, B)=\frac{1}{2}\left\|\log \left(B^{-1 / 2} A B^{-1 / 2}\right)\right\|_{F}^{2},
$$

where $\log (A)$ denotes the principal logarithm of the matrix $A$ and \|\|$_{F}$ denotes the Frobenius norm. This is the geodesic distance associated with the so-called affine-invariant Riemannian metric $[5,6,4,7,8]$.

2. The Alpha Log-Det divergences [9], corresponding to $D^{(\alpha, 1-\alpha)}(A, B)$, with

$$
\begin{aligned}
D^{(\alpha, 1-\alpha)}(A, B) & =\frac{1}{\alpha(1-\alpha)} \log \left[\frac{\operatorname{det}[\alpha A+(1-\alpha) B]}{\operatorname{det}(A)^{\alpha} \operatorname{det}(B)^{1-\alpha}}\right], 0<\alpha<1, \\
D^{(1,0)}(A, B) & =\operatorname{tr}\left(A^{-1} B-I\right)-\log \operatorname{det}\left(A^{-1} B\right), \\
D^{(0,1)}(A, B) & =\operatorname{tr}\left(B^{-1} A-I\right)-\log \operatorname{det}\left(B^{-1} A\right) .
\end{aligned}
$$

The case $\alpha=1 / 2$ gives the symmetric Stein divergence (also called the JensenBregman LogDet divergence), whose square root is a metric on $\mathrm{Sym}^{++}(n)$ [10], with $D^{(1 / 2,1 / 2)}(A, B)=4 d_{\text {stein }}^{2}(A, B)=4\left[\log \operatorname{det}\left(\frac{A+B}{2}\right)-\frac{1}{2} \log \operatorname{det}(A B)\right]$.

Previous work. In [1], we generalized the Alpha Log-Det divergences between SPD matrices [9] to the infinite-dimensional Alpha Log-Determinant divergences between positive definite unitized trace class operators on an infinite-dimensional Hilbert space. This is done via the introduction of the extended Fredholm determinant for 
unitized trace class operators, along with the corresponding generalization of the logconcavity of the determinant for SPD matrices to the infinite-dimensional setting. In [2], we present a formulation for the Alpha-Beta Log-Det divergences between positive definite unitized trace class operators, generalizing the Alpha-Beta Log-Det divergences between SPD matrices as defined by Eq.(1). In both [1] and [2], for the divergences between reproducing kernel Hilbert spaces (RKHS) covariance operators, we obtain closed form formulas for the Alpha-Beta Log-Det divergences via the corresponding Gram matrices.

Contributions of this work. The current work is a continuation and generalization of [1] and [2]. In particular, we generalize the Alpha-Beta Log-Det divergences in [2] to the entire Hilbert manifold of positive definite unitized Hilbert-Schmidt operators on an infinite-dimensional Hilbert space. This is done by the introduction of the extended Hilbert-Carleman determinant for unitized Hilbert-Schmidt operators, in addition to the extended Fredholm determinant for unitized trace class operators employed in [1] and [2]. As in the finite-dimensional setting [3] and in [1], [2], the resulting family of divergences is general and admits as special cases many metrics and distance-like functions between positive definite unitized Hilbert-Schmidt operators, including the infinite-dimensional affine-invariant Riemannian distance in [11].

Comparison with the formulations in [1] and [2]. While the mathematical formulation presented in the current work, for Hilbert-Schmidt operators, is more general than the formulations in [1] and [2], which are for trace class operators, it should not be considered as a substitute for them. Many results in [1] and [2], especially those involving covariance operators, require explicitly the trace class assumption.

\section{Positive definite unitized trace class and Hilbert-Schmidt operators}

Throughout the paper, we assume that $\mathcal{H}$ is a real separable Hilbert space, with $\operatorname{dim}(\mathcal{H})=\infty$, unless explicitly stated otherwise. Let $\mathcal{L}(\mathcal{H})$ be the Banach space of bounded linear operators on $\mathcal{H}$, with operator norm \|\| . Let $\operatorname{Sym}(\mathcal{H}) \subset \mathcal{L}(\mathcal{H})$ denote the subspace of bounded, self-adjoint operators on $\mathcal{H}$. Let $\operatorname{Sym}^{+}(\mathcal{H}) \subset \operatorname{Sym}(\mathcal{H})$ denote the set of self-adjoint, positive operators on $\mathcal{H}$, that is $A \in \operatorname{Sym}^{+}(\mathcal{H}) \Longleftrightarrow$ 
$\langle x, A x\rangle \geq 0 \forall x \in \mathcal{H}$. Let $\operatorname{Sym}^{++}(\mathcal{H}) \subset \operatorname{Sym}^{+}(\mathcal{H})$ denote the set of self-adjoint, strictly positive operators on $\mathcal{H}$, that is $A \in \operatorname{Sym}^{++}(\mathcal{H}) \Longleftrightarrow\langle x, A x\rangle>0 \forall x \in$ $\mathcal{H}, x \neq 0$, or equivalently, $\operatorname{ker}(A)=\{0\}$.

Most importantly, we consider the set $\mathbb{P}(\mathcal{H}) \subset \operatorname{Sym}^{++}(\mathcal{H})$ of self-adjoint, bounded, positive definite operators on $\mathcal{H}$, which is defined by

$$
A \in \mathbb{P}(\mathcal{H}) \Longleftrightarrow A=A^{*}, \exists M_{A}>0 \text { such that }\langle x, A x\rangle \geq M_{A} \|\left. x\right|^{2} \quad \forall x \in \mathcal{H} .
$$

We use the notation $A>0 \Longleftrightarrow A \in \mathbb{P}(\mathcal{H})$.

In the following, let $\mathscr{C}_{p}(\mathcal{H})$ denote the set of $p$ th Schatten class operators on $\mathcal{H}$ (see e.g. [12]), under the norm \|\|$_{p}, 1 \leq p \leq \infty$, which is defined by

$$
\mathscr{C}_{p}(\mathcal{H})=\left\{A \in \mathcal{L}(\mathcal{H}):\|A\|_{p}=\left(\operatorname{tr}|A|^{p}\right)^{1 / p}<\infty\right\},
$$

where $|A|=\left(A^{*} A\right)^{1 / 2}$.

The cases we consider in this work are: (i) the space $\mathscr{C}_{1}(\mathcal{H})$ of trace class operators on $\mathcal{H}$, which we also denote by $\operatorname{Tr}(\mathcal{H})$, and (ii) the space $\mathscr{C}_{2}(\mathcal{H})$ of Hilbert-Schmidt operators on $\mathcal{H}$, which we also denote by $\operatorname{HS}(\mathcal{H})$.

Extended (unitized) trace class operators. In [1], we define the set of extended (or unitized) trace class operators on $\mathcal{H}$ to be

$$
\operatorname{Tr}_{X}(\mathcal{H})=\{A+\gamma I: A \in \operatorname{Tr}(\mathcal{H}), \gamma \in \mathbb{R}\}
$$

The set $\operatorname{Tr}_{X}(\mathcal{H})$ becomes a Banach algebra under the extended trace class norm

$$
\|A+\gamma I\|_{\operatorname{tr}_{X}}=\|A\|_{\operatorname{tr}}+|\gamma|=\operatorname{tr}|A|+|\gamma| .
$$

For $(A+\gamma I) \in \operatorname{Tr}_{X}(\mathcal{H})$, its extended trace is defined to be

$$
\operatorname{tr}_{X}(A+\gamma I)=\operatorname{tr}(A)+\gamma .
$$

By this definition $\operatorname{tr}_{X}(I)=1$, in contrast to standard trace definition, according to which $\operatorname{tr}(I)=\infty$.

Extended (unitized) Hilbert-Schmidt operators. In [11], the author considered the following set of extended (unitized) Hilbert-Schmidt operators

$$
\operatorname{HS}_{X}(\mathcal{H})=\{A+\gamma I: A \in \operatorname{HS}(\mathcal{H}), \gamma \in \mathbb{R}\}
$$


The set $\operatorname{HS}_{X}(\mathcal{H})$ can be equipped with the extended Hilbert-Schmidt inner product $\langle,\rangle_{\mathrm{eHS}}$, defined by

$$
\langle A+\gamma I, B+\mu I\rangle_{\mathrm{eHS}}=\langle A, B\rangle_{\mathrm{HS}}+\gamma \mu=\operatorname{tr}\left(A^{*} B\right)+\gamma \mu .
$$

along with the associated extended Hilbert-Schmidt norm

$$
\|A+\gamma I\|_{\mathrm{eHS}}^{2}=\|A\|_{\mathrm{HS}}^{2}+\gamma^{2}=\operatorname{tr}\left(A^{*} A\right)+\gamma^{2}
$$

Under the inner product $\langle,\rangle_{\mathrm{eHS}}$, the Hilbert-Schmidt operators are orthogonal to the scalar operators. Under the norm \|\|$_{\mathrm{eHS}},\|I\|_{\mathrm{eHS}}=1$, in contrast to the standard Hilbert-Schmidt norm, according to which $\|I\|_{\mathrm{HS}}=\infty$.

Positive definite unitized trace class and Hilbert-Schmidt operators. The set of positive definite unitized trace class operators $\mathscr{P} \mathscr{C}_{1}(\mathcal{H}) \subset \operatorname{Tr}_{X}(\mathcal{H})$ is defined to be the intersection

$$
\mathscr{P} \mathscr{C}_{1}(\mathcal{H})=\operatorname{Tr}_{X}(\mathcal{H}) \cap \mathbb{P}(\mathcal{H})=\left\{A+\gamma I>0: A^{*}=A, A \in \operatorname{Tr}(\mathcal{H}), \gamma \in \mathbb{R}\right\}
$$

The set of positive definite unitized Hilbert-Schmidt operators $\mathscr{P} \mathscr{C}_{2}(\mathcal{H}) \subset \operatorname{HS}_{X}(\mathcal{H})$ is defined to be the intersection

$$
\mathscr{P} \mathscr{C}_{2}(\mathcal{H})=\operatorname{HS}_{X}(\mathcal{H}) \cap \mathbb{P}(\mathcal{H})=\left\{A+\gamma I>0: A=A^{*}, A \in \operatorname{HS}(\mathcal{H}), \gamma \in \mathbb{R}\right\} .
$$

Remark 1. In [1] and [2], we use the notations $\operatorname{PTr}(\mathcal{H})$ and $\Sigma(\mathcal{H})$ to denote $\mathscr{P} \mathscr{C}_{1}(\mathcal{H})$ and $\mathscr{P} \mathscr{C}_{2}(\mathcal{H})$, respectively. In the following, we refer to elements of $\mathscr{P} \mathscr{C}_{1}(\mathcal{H})$ and $\mathscr{P} \mathscr{C}_{2}(\mathcal{H})$ as positive definite trace class operators and positive definite Hilbert-Schmidt operators, respectively.

In [11], it is shown that the set $\mathscr{P} \mathscr{C}_{2}(\mathcal{H})$ assumes the structure of an infinitedimensional Hilbert manifold and can be equipped with the following Riemannian metric. For each $P \in \mathscr{P} \mathscr{C}_{2}(\mathcal{H})$, on the tangent space $T_{P}\left(\mathscr{P} \mathscr{C}_{2}(\mathcal{H})\right) \cong \mathcal{H}_{\mathbb{R}}=\{A+\gamma I$ : $\left.A=A^{*}, A \in \operatorname{HS}(\mathcal{H}), \gamma \in \mathbb{R}\right\}$, we define the following inner product

$$
\langle A+\gamma I, B+\mu I\rangle_{P}=\left\langle P^{-1 / 2}(A+\gamma I) P^{-1 / 2}, P^{-1 / 2}(B+\mu I) P^{-1 / 2}\right\rangle_{\mathrm{eHS}} .
$$


The Riemannian metric given by $\langle,\rangle_{P}$ then makes $\mathscr{P} \mathscr{C}_{2}(\mathcal{H})$ an infinite-dimensional Riemannian manifold. Under this Riemannian metric, the geodesic distance between $(A+\gamma I),(B+\mu I) \in \mathscr{P} \mathscr{C}_{2}(\mathcal{H})$ is given by

$$
d_{\mathrm{aiHS}}[(A+\gamma I),(B+\mu I)]=\left\|\log \left[(B+\mu I)^{-1 / 2}(A+\gamma I)(B+\mu I)^{-1 / 2}\right]\right\|_{\mathrm{eHS}} .
$$

Aim of this work. In [1], we introduce a parametrized family of divergences, called Log-Determinant divergences, between operators in $\mathscr{P} \mathscr{C}_{1}(\mathcal{H})$. In [2], we generalize these to the Alpha-Beta Log-Determinant divergences on $\mathscr{P} \mathscr{C}_{1}(\mathcal{H})$, which include the distance $d_{\text {aiHs }}$ as a special case. However, these divergences are defined specifically on $\mathscr{P} \mathscr{C}_{1}(\mathcal{H})$. In the case $\operatorname{dim}(\mathcal{H})=\infty$, the set $\mathscr{P} \mathscr{C}_{1}(\mathcal{H})$ of positive definite trace class operators on $\mathcal{H}$ is a strict subset of the set of positive definite Hilbert-Schmidt operators $\mathscr{P} \mathscr{C}_{2}(\mathcal{H})$. In this work, we generalize the divergences in [1] and [2] to all of $\mathscr{P} \mathscr{C}_{2}(\mathcal{H})$.

\section{Functions of positive definite unitized Hilbert-Schmidt operators}

We first discuss several important functions on $\mathscr{P} \mathscr{C}_{2}(\mathcal{H})$, namely the exponential, logarithm, and power functions.

Exponential and logarithm functions. Consider the exponential function exp : $\mathcal{L}(\mathcal{H}) \rightarrow \mathcal{L}(\mathcal{H})$ defined by

$$
\exp (A)=\sum_{k=0}^{\infty} \frac{A^{k}}{k !}
$$

In [11], it is shown that the map exp : $\operatorname{Sym}(\mathcal{H}) \cap \mathrm{HS}_{X}(\mathcal{H}) \rightarrow \mathscr{P} \mathscr{C}_{2}(\mathcal{H})$ and its inverse function $\log =\exp ^{-1}: \mathscr{P} \mathscr{C}_{2}(\mathcal{H}) \rightarrow \operatorname{Sym}(\mathcal{H}) \cap \mathrm{HS}_{X}(\mathcal{H})$ are diffeomorphisms. Here, for any $(A+\gamma I) \in \mathscr{P} \mathscr{C}_{2}(\mathcal{H}), \log (A+\gamma I)$ is defined via the spectral decomposition of $A$ as follows. Let $\left\{\lambda_{k}\right\}_{k=1}^{\infty}$ be the eigenvalues of $A$ with corresponding orthonormal eigenvectors $\left\{\phi_{k}\right\}_{k=1}^{\infty}$. Then

$$
A=\sum_{k=1}^{\infty} \lambda_{k} \phi_{k} \otimes \phi_{k}, \quad \log (A+\gamma I)=\sum_{k=1}^{\infty} \log \left(\lambda_{k}+\gamma\right) \phi_{k} \otimes \phi_{k}
$$


where $\phi_{k} \otimes \phi_{k}: \mathcal{H} \rightarrow \mathcal{H}$ is a rank-one operator defined by $\left(\phi_{k} \otimes \phi_{k}\right) w=\left\langle\phi_{k}, w\right\rangle \phi_{k}$ $\forall w \in \mathcal{H}$. Since $\log (A+\gamma I) \in \operatorname{Sym}(\mathcal{H}) \cap \operatorname{HS}_{X}(\mathcal{H})$, it has the form

$$
\log (A+\gamma I)=A_{1}+\gamma_{1} I, \quad A_{1} \in \operatorname{Sym}(\mathcal{H}) \cap \operatorname{HS}(\mathcal{H}), \gamma_{1} \in \mathbb{R} .
$$

Power functions. Given the exponential and logarithm functions, for any $\alpha \in \mathbb{R}$, the power function $(A+\gamma I)^{\alpha}$, for $(A+\gamma I) \in \mathscr{P} \mathscr{C}_{2}(\mathcal{H})$, is then well-defined via the following expression

$$
(A+\gamma I)^{\alpha}=\exp [\alpha \log (A+\gamma I)] \in \mathscr{P} \mathscr{C}_{2}(\mathcal{H}) .
$$

Furthermore, for any two operators $(A+\gamma I),(B+\mu I) \in \mathscr{P} \mathscr{C}_{2}(\mathcal{H})$, we show that

$$
\log \left[(A+\gamma I)(B+\mu I)^{-1}\right], \quad\left[(A+\gamma I)(B+\mu I)^{-1}\right]^{\alpha}, \alpha \in \mathbb{R}
$$

are all well-defined and are elements of $\operatorname{HS}_{X}(\mathcal{H})$ (though not necessarily of $\operatorname{Sym}(\mathcal{H})$ ). To this end, let $B \in \mathcal{L}(\mathcal{H})$ be any invertible operator, then for any $A \in \mathcal{L}(\mathcal{H})$, we have

$$
\exp \left(B A B^{-1}\right)=\sum_{j=0}^{\infty} \frac{\left(B A B^{-1}\right)^{j}}{j !}=B\left(\sum_{j=0}^{\infty} \frac{A^{j}}{j !}\right) B^{-1}=B \exp (A) B^{-1} .
$$

Thus for $(A+\gamma I) \in \mathscr{P} \mathscr{C}_{2}(\mathcal{H})$, the logarithm of $B(A+\gamma I) B^{-1}=B A B^{-1}+\gamma I \in$ $\operatorname{HS}_{X}(\mathcal{H})$ is also well-defined and is given by

$$
\begin{aligned}
\log \left[B(A+\gamma I) B^{-1}\right] & =B \log (A+\gamma I) B^{-1} \\
& =B\left(A_{1}+\gamma_{1} I\right) B^{-1}=B A_{1} B^{-1}+\gamma_{1} I \in \mathrm{HS}_{X}(\mathcal{H}) .
\end{aligned}
$$

Using Eq. (15), we obtain the following results.

Proposition 1. Let $(A+\gamma I),(B+\mu I) \in \mathscr{P} \mathscr{C}_{2}(\mathcal{H})$. Let $\Lambda+\frac{\gamma}{\mu} I=(B+\mu I)^{-1 / 2}(A+$ $\gamma I)(B+\mu I)^{-1 / 2}$. Then

1. The logarithm function $\log \left[(A+\gamma I)(B+\mu I)^{-1}\right] \in \operatorname{HS}_{X}(\mathcal{H})$ is well-defined and is given by

$$
\log \left[(A+\gamma I)(B+\mu I)^{-1}\right]=(B+\mu I)^{1 / 2} \log \left(\Lambda+\frac{\gamma}{\mu} I\right)(B+\mu I)^{-1 / 2} .
$$


2. For any $\alpha \in \mathbb{R}$, the power function $\left[(A+\gamma I)(B+\mu I)^{-1}\right]^{\alpha} \in \mathrm{HS}_{X}(\mathcal{H})$ is well-defined and is given by

$$
\left[(A+\gamma I)(B+\mu I)^{-1}\right]^{\alpha}=(B+\mu I)^{1 / 2}\left(\Lambda+\frac{\gamma}{\mu} I\right)^{\alpha}(B+\mu I)^{-1 / 2} .
$$

\section{The extended Hilbert-Carleman determinant}

The key concept for defining Log-Determinant divergences between operators is determinant. We recall that for $A \in \operatorname{Tr}(\mathcal{H})$, the Fredholm determinant $\operatorname{det}(I+A)$ is (see e.g. [13])

$$
\operatorname{det}(I+A)=\prod_{k=1}^{\infty}\left(1+\lambda_{k}\right)
$$

where $\left\{\lambda_{k}\right\}_{k=1}^{\infty}$ are the eigenvalues of $A$. To define Log-Determinant divergences between positive definite trace class operators in $\mathscr{P} \mathscr{C}_{1}(\mathcal{H})$, in [1], we generalize the Fredholm determinant to the extended Fredholm determinant of extended trace class operators. For $(A+\gamma I) \in \operatorname{Tr}_{X}(\mathcal{H}), \gamma \neq 0$, its extended Fredholm determinant is defined to be, assuming that $\operatorname{dim}(\mathcal{H})=\infty$,

$$
\operatorname{det}_{\mathrm{X}}(A+\gamma I)=\frac{1}{\gamma} \operatorname{det}\left(\frac{A}{\gamma}+I\right)
$$

where the determinant on the right hand side is the Fredholm determinant (we refer to [1] for the derivation leading to this definition). For $\gamma=1$, we recover the Fredholm determinant. In the case $\operatorname{dim}(\mathcal{H})<\infty$, we $\operatorname{define} \operatorname{det}_{\mathrm{X}}(A+\gamma I)=\operatorname{det}(A+\gamma I)$, the standard matrix determinant.

The extended Fredholm determinant continues to play a key role in the current work, but it is not sufficient for dealing with positive definite Hilbert-Schmidt operators in $\mathscr{P} \mathscr{C}_{2}(\mathcal{H})$. In order to do so, we introduce the concept of extended Hilbert-Carleman determinant.

We first recall the concept of the Hilbert-Carleman determinant for operators of the form $I+A$, where $A$ is a Hilbert-Schmidt operator (see e.g. [13] for a comprehensive treatment). Following [13], for any bounded operator $A \in \mathcal{L}(\mathcal{H})$, consider the operator

$$
R_{n}(A)=\left[(I+A) \exp \left(\sum_{k=1}^{n-1} \frac{(-A)^{k}}{k}\right)\right]-I .
$$


If $A \in \mathscr{C}_{n}(\mathcal{H})$, then $R_{n}(A) \in \mathscr{C}_{1}(\mathcal{H})$. Thus the following quantity is well-defined

$$
\operatorname{det}_{\mathrm{n}}(I+A)=\operatorname{det}\left(I+R_{n}(A)\right) \text {. }
$$

In particular, for $n=1$, we obtain $R_{1}(A)=A$ and thus

$$
\operatorname{det}_{1}(I+A)=\operatorname{det}(I+A)
$$

For $n=2$, we have $R_{2}(A)=(I+A) \exp (-A)-I$ and thus

$$
\operatorname{det}_{2}(I+A)=\operatorname{det}[(I+A) \exp (-A)]
$$

This is called the Hilbert-Carleman determinant of $I+A$. In particular, for $A \in$ $\operatorname{Tr}(\mathcal{H})=\mathscr{C}_{1}(\mathcal{H})$, we have

$$
\begin{aligned}
\operatorname{det}_{2}(I+A) & =\operatorname{det}(I+A) \exp (-\operatorname{tr}(A)), \\
\log \operatorname{det}_{2}(I+A) & =\log \operatorname{det}(I+A)-\operatorname{tr}(A) .
\end{aligned}
$$

The function $\operatorname{det}_{2}(I+A)$ is continuous in the Hilbert-Schmidt norm, so that

$$
\lim _{k \rightarrow \infty}\left\|A_{k}-A\right\|_{\mathrm{HS}}=0 \Rightarrow \lim _{k \rightarrow \infty} \operatorname{det}_{2}\left(I+A_{k}\right)=\operatorname{det}_{2}(I+A) .
$$

We first have the following result.

Lemma 1. Assume that $A \in \operatorname{Sym}(\mathcal{H}) \cap \operatorname{HS}(\mathcal{H})$ such that $I+A>0$. Let $\left\{\lambda_{k}\right\}_{k=1}^{\infty}$ be the eigenvalues of $A$. Then

$$
\log \operatorname{det}_{2}(I+A)=\sum_{k=1}^{\infty}\left[\log \left(1+\lambda_{k}\right)-\lambda_{k}\right]
$$

is well-defined and finite. Furthermore,

$$
\log \operatorname{det}_{2}(I+A) \leq 0
$$

with equality if and only if $A=0$.

The Hilbert-Carleman determinant $\operatorname{det}_{2}$ is defined for operators of the form $A+I$, $A \in \operatorname{HS}(\mathcal{H})$, but not for operators of the form $A+\gamma I, \gamma>0, \gamma \neq 1$. In the following, we generalize $\operatorname{det}_{2}$ to handle these operators. We first have the following generalization of the function $R_{2}(A)=(I+A) \exp (-A)-I$ above. 
Lemma 2. Assume that $(A+\gamma I) \in \operatorname{HS}_{X}(\mathcal{H}), \gamma \neq 0$. Define

$$
R_{2, \gamma}(A)=(A+\gamma I) \exp (-A / \gamma)-\gamma I
$$

Then $R_{2, \gamma}(A) \in \operatorname{Tr}(\mathcal{H})$ and hence $R_{2, \gamma}(A)+\gamma I=(A+\gamma I) \exp (-A / \gamma) \in \operatorname{Tr}_{X}(\mathcal{H})$. This also implies that the infinite product

$$
\prod_{k=1}^{\infty}\left[\left(\lambda_{k}+\gamma\right) \exp \left(-\lambda_{k} / \gamma\right)-\gamma+1\right]
$$

converges to a finite value, where $\left\{\lambda_{k}\right\}_{k=1}^{\infty}$ are the eigenvalues of $A$.

In particular, for $\gamma=1$, we have $R_{2,1}(A)=R_{2}(A)$. Motivated by Lemma 2 and the definition of $\operatorname{det}_{2}$, we arrive at the following generalization of $\operatorname{det}_{2}$.

Definition 1 (Extended Hilbert-Carleman determinant). For $(A+\gamma I) \in \operatorname{HS}_{X}(\mathcal{H})$, $\gamma \neq 0$, its extended Hilbert-Carleman determinant is defined to be

$$
\operatorname{det}_{2 \mathrm{X}}(A+\gamma I)=\operatorname{det}_{\mathrm{X}}\left[R_{2, \gamma}(A)+\gamma I\right]=\operatorname{det}_{\mathrm{X}}[(A+\gamma I) \exp (-A / \gamma)]
$$

If $\gamma=1$, then we recover the Hilbert-Carleman determinant

$$
\operatorname{det}_{2 \mathrm{X}}(A+I)=\operatorname{det}[(A+I) \exp (-A)]=\operatorname{det}_{2}(A+I) .
$$

If $(A+\gamma I) \in \operatorname{Tr}_{X}(\mathcal{H})$, then

$$
\operatorname{det}_{2 \mathrm{X}}(A+\gamma I)=\operatorname{det}_{\mathrm{X}}(A+\gamma I) \exp (-\operatorname{tr}(A) / \gamma) .
$$

The following are the some of the properties of $\operatorname{det}_{2 \mathrm{x}}$ which we employ later on.

\section{Lemma 3 (Factorization Rule).}

$$
\operatorname{det}_{2 \mathrm{X}}(A+\gamma I)=\gamma \operatorname{det}_{2}\left(\frac{A}{\gamma}+I\right) .
$$

If $(A+\gamma I) \in \operatorname{Tr}_{X}(\mathcal{H}), \gamma \neq 0$, then Lemma 5 in [1] states that for any invertible operator $C \in \mathcal{L}(\mathcal{H})$, we have

$$
\operatorname{det}_{\mathrm{X}}\left[C(A+\gamma I) C^{-1}\right]=\operatorname{det}_{\mathrm{X}}(A+\gamma I)
$$

This property generalizes for $\operatorname{det}_{2 \mathrm{X}}$, with $(A+\gamma I) \in \mathrm{HS}_{X}(\mathcal{H})$, as follows. 
Lemma 4 (Similarity Invariant). Let $(A+\gamma I) \in \operatorname{HS}_{X}(\mathcal{H}), \gamma \neq 0$. Let $C \in \mathcal{L}(\mathcal{H})$ be invertible. Then

$$
\operatorname{det}_{2 \mathrm{X}}\left[C(A+\gamma I) C^{-1}\right]=\operatorname{det}_{2 \mathrm{X}}(A+\gamma I)
$$

For $(A+\gamma I),(B+\mu I) \in \operatorname{Tr}_{X}(\mathcal{H})$, we show in Proposition 4 in [1] that the product rule for determinants holds, that is $\operatorname{det}_{\mathrm{X}}[(A+\gamma I)(B+\mu I)]=\operatorname{det}_{\mathrm{X}}(A+$ $\gamma I) \operatorname{det}_{\mathrm{X}}(B+\mu I)$. For $\operatorname{det}_{2}$ and $\operatorname{det}_{2 \mathrm{X}}$ and $(A+\gamma I),(B+\mu I) \in \mathrm{HS}_{X}(\mathcal{H})$, this is no longer true in general. However, if $(A+\gamma I),(B+\mu I) \in \mathscr{P} \mathscr{C}_{2}(\mathcal{H})$, or if $(A+\gamma I),(B+\mu I) \in \operatorname{Tr}_{X}(\mathcal{H})$, then we still have commutativity, that is $\operatorname{det}_{2 \mathrm{X}}[(A+$ $\gamma I)(B+\mu I)]=\operatorname{det}_{2 \mathrm{X}}[(B+\mu I)(A+\gamma I)]$, as follows.

Lemma 5 (Commutativity). Assume that $(A+\gamma I),(B+\mu I) \in \mathscr{P} \mathscr{C}_{2}(\mathcal{H})$. Then

$$
\begin{aligned}
\operatorname{det}_{2 \mathrm{X}}[(A+\gamma I)(B+\mu I)] & =\operatorname{det}_{2 \mathrm{X}}\left[(A+\gamma I)^{1 / 2}(B+\mu I)(A+\gamma I)^{1 / 2}\right] \\
& =\operatorname{det}_{2 \mathrm{X}}[(B+\mu I)(A+\gamma I)] \\
& =\operatorname{det}_{2 \mathrm{X}}\left[(B+\mu I)^{1 / 2}(A+\gamma I)(B+\mu I)^{1 / 2}\right]
\end{aligned}
$$

If $(A+\gamma I),(B+\mu I) \in \operatorname{Tr}_{X}(\mathcal{H}), \gamma \neq 0, \mu \neq 0$, then

$$
\operatorname{det}_{2 \mathrm{X}}[(A+\gamma I)(B+\mu I)]=\operatorname{det}_{2 \mathrm{X}}[(B+\mu I)(A+\gamma I)] \text {. }
$$

An immediate consequence of Lemma 5 is the following.

Corollary 1 (Cyclic Property). Assume that $(A+\gamma I),(B+\mu I),(C+\nu I) \in \mathscr{P} \mathscr{C}_{2}(\mathcal{H})$, or $(A+\gamma I),(B+\mu I),(C+\nu I) \in \operatorname{Tr}_{X}(\mathcal{H})$. Then

$$
\begin{aligned}
\operatorname{det}_{2 \mathrm{X}}[(A+\gamma I)(B+\mu I)(C+\nu I)] & =\operatorname{det}_{2 \mathrm{X}}[(C+\nu I)(A+\gamma I)(B+\mu I)] \\
& =\operatorname{det}_{2 \mathrm{X}}[(B+\mu I)(C+\nu I)(A+\gamma I)] .
\end{aligned}
$$

For the following properties, we assume explicitly that $(A+\gamma I),(B+\mu I) \in$ $\mathscr{P} \mathscr{C}_{2}(\mathcal{H})$, that is $(A+\gamma I)>0,(B+\mu I)>0$ and $A, B \in \mathrm{HS}(\mathcal{H})$. These properties are utilized in the formulation of the Log-Determinant divergences in Section 5. 
Lemma 6. Let $(A+\gamma I),(B+\mu I) \in \mathscr{P} \mathscr{C}_{2}(\mathcal{H})$. Let $\Lambda+\nu I=(B+\mu I)^{-1 / 2}(A+$ $\gamma I)(B+\mu I)^{-1 / 2}$, where $\Lambda \in \operatorname{HS}(\mathcal{H})$ and $\nu=\frac{\gamma}{\mu}$. Then for any $\alpha \in \mathbb{R}$,

$$
\begin{aligned}
\operatorname{det}_{2 \mathrm{X}}\left(\left[(A+\gamma I)(B+\mu I)^{-1}\right]^{\alpha}\right) & =\operatorname{det}_{2 \mathrm{X}}\left[(\Lambda+\nu I)^{\alpha}\right] \\
& =\operatorname{det}_{2 \mathrm{X}}\left(\left[(B+\mu I)^{-1}(A+\gamma I)\right]^{\alpha}\right) .
\end{aligned}
$$

Lemma 7. Let $(I+A) \in \mathscr{P} \mathscr{C}_{2}(\mathcal{H})$. Let $\alpha \in \mathbb{R}$ be arbitrary. Then $(I+A)^{\alpha}-I \in$ $\operatorname{Sym}(\mathcal{H}) \cap \operatorname{HS}(\mathcal{H})$. Let $\left\{\lambda_{k}\right\}_{k=1}^{\infty}$ be the eigenvalues of $A$. Then the following quantities converge to finite values:

$$
\begin{aligned}
\operatorname{det}_{2}\left[(I+A)^{\alpha}\right] & =\prod_{k=1}^{\infty}\left(1+\lambda_{k}\right)^{\alpha} \exp \left[1-\left(1+\lambda_{k}\right)^{\alpha}\right] \\
\log \operatorname{det}_{2}\left[(I+A)^{\alpha}\right] & =\sum_{k=1}^{\infty}\left[\alpha \log \left(1+\lambda_{k}\right)+1-\left(1+\lambda_{k}\right)^{\alpha}\right] .
\end{aligned}
$$

Furthermore,

$$
\log \operatorname{det}_{2}\left[(I+A)^{\alpha}\right] \leq 0
$$

with equality if and only if $A=0$. For $(I+A) \in \mathscr{P} \mathscr{C}_{1}(\mathcal{H})$,

$$
\begin{aligned}
\operatorname{det}_{2}\left[(I+A)^{\alpha}\right] & =\operatorname{det}\left[(I+A)^{\alpha}\right] \exp \left(-\operatorname{tr}\left[(I+A)^{\alpha}-I\right]\right), \\
\log \operatorname{det}_{2}\left[(I+A)^{\alpha}\right] & =\alpha \log \operatorname{det}(I+A)-\operatorname{tr}\left[(I+A)^{\alpha}-I\right] .
\end{aligned}
$$

Lemma 8. Let $(A+\gamma I) \in \mathscr{P} \mathscr{C}_{2}(\mathcal{H})$. Let $\alpha \in \mathbb{R}$ be arbitrary. Then $(A+\gamma I)^{\alpha}-\gamma^{\alpha} I \in$ $\operatorname{Sym}(\mathcal{H}) \cap \operatorname{HS}(\mathcal{H})$. Let $\left\{\lambda_{k}\right\}_{k=1}^{\infty}$ be the eigenvalues of $A$. Then the following quantities converge to finite values:

$$
\begin{aligned}
\operatorname{det}_{2 \mathrm{X}}\left[(A+\gamma I)^{\alpha}\right] & =\gamma^{\alpha} \prod_{k=1}^{\infty}\left(1+\frac{\lambda_{k}}{\gamma}\right)^{\alpha} \exp \left[1-\left(1+\frac{\lambda_{k}}{\gamma}\right)^{\alpha}\right] \\
\log \operatorname{det}_{2 \mathrm{X}}\left[(A+\gamma I)^{\alpha}\right] & =\alpha \log \gamma+\sum_{k=1}^{\infty}\left[\alpha \log \left(1+\frac{\lambda_{k}}{\gamma}\right)+1-\left(1+\frac{\lambda_{k}}{\gamma}\right)^{\alpha}\right]
\end{aligned}
$$




$$
\begin{aligned}
& \text { For }(A+\gamma I) \in \mathscr{P}_{\mathscr{C}_{1}(\mathcal{H})} \\
& \begin{aligned}
\operatorname{det}_{2 \mathrm{X}}\left[(A+\gamma I)^{\alpha}\right] & =\gamma^{\alpha} \operatorname{det}\left[\left(\frac{A}{\gamma}+I\right)^{\alpha}\right] \exp \left(-\operatorname{tr}\left[\left(\frac{A}{\gamma}+I\right)^{\alpha}-I\right]\right) \\
\log \operatorname{det}_{2 \mathrm{X}}\left[(A+\gamma I)^{\alpha}\right] & =\alpha \log \gamma+\alpha \log \operatorname{det}\left(\frac{A}{\gamma}+I\right)-\operatorname{tr}\left[\left(\frac{A}{\gamma}+I\right)^{\alpha}-I\right] \\
& =\alpha \log \operatorname{det}(A+\gamma I)-\operatorname{tr}\left[\left(\frac{A}{\gamma}+I\right)^{\alpha}-I\right]
\end{aligned}
\end{aligned}
$$

\section{Infinite-dimensional Log-Determinant divergences between positive definite Hilbert-}

\section{Schmidt operators}

In [2], we define the Log-Determinant divergences between two positive definite trace class operators $(A+\gamma I),(B+\mu I) \in \mathscr{P} \mathscr{C}_{1}(\mathcal{H})$ as follows

$$
\begin{aligned}
& D_{r}^{(\alpha, \beta)}[(A+\gamma I),(B+\mu I)] \\
& =\frac{1}{\alpha \beta} \log \left[\left(\frac{\gamma}{\mu}\right)^{r\left(\delta-\frac{\alpha}{\alpha+\beta}\right)} \operatorname{det}_{\mathrm{X}}\left(\frac{\alpha\left(\Lambda+\frac{\gamma}{\mu} I\right)^{r(1-\delta)}+\beta\left(\Lambda+\frac{\gamma}{\mu} I\right)^{-r \delta}}{\alpha+\beta}\right)\right] .
\end{aligned}
$$

Here $\alpha>0, \beta>0, r \neq 0$ are fixed, $\Lambda+\frac{\gamma}{\mu} I=(B+\mu I)^{-1 / 2}(A+\gamma I)(B+\mu I)^{-1 / 2}$, and $\delta=\frac{\alpha \gamma^{r}}{\alpha \gamma^{r}+\beta \mu^{r}}$. This definition is motivated by the infinite-dimensional generalizations of Ky Fan's inequality [14] on the log-concavity of the determinant of SPD matrices, as stated for $\operatorname{det}_{\mathrm{X}}$ in Theorem 1 in [1] and Theorem 5 in [2].

In the following, we show that the definition given in Eq. (52) is valid in the more general case $(A+\gamma I),(B+\mu I) \in \mathscr{P} \mathscr{C}_{2}(\mathcal{H})$. We first have the following results.

Proposition 2. Assume that $(A+\gamma I),(B+\mu I) \in \mathscr{P} \mathscr{C}_{2}(\mathcal{H})$. Let $\alpha>0, \beta>0$ be fixed. Let $p, q \in \mathbb{R}$ be such that $p \alpha(\gamma / \mu)^{p}=q \beta(\gamma / \mu)^{-q}$. Then for $\Lambda+\frac{\gamma}{\mu} I=$ $(B+\mu I)^{-1 / 2}(A+\gamma I)(B+\mu I)^{-1 / 2}$,

$$
\frac{\alpha\left(\Lambda+\frac{\gamma}{\mu} I\right)^{p}+\beta\left(\Lambda+\frac{\gamma}{\mu} I\right)^{-q}}{\alpha+\beta} \in \mathscr{P} \mathscr{C}_{1}(\mathcal{H}) .
$$

Proposition 3. Assume that $(A+\gamma I),(B+\mu I) \in \mathscr{P} \mathscr{C}_{2}(\mathcal{H})$. Let $\Lambda+\frac{\gamma}{\mu} I=(B+$ $\mu I)^{-1 / 2}(A+\gamma I)(B+\mu I)^{-1 / 2}$. Let $\alpha>0, \beta>0$ be fixed. Let $p, q \in \mathbb{R}$ be such that $p \alpha(\gamma / \mu)^{p}=q \beta(\gamma / \mu)^{-q}$. Then

$$
\frac{\alpha\left[(A+\gamma I)(B+\mu I)^{-1}\right]^{p}+\beta\left[(A+\gamma I)(B+\mu I)^{-1}\right]^{-q}}{\alpha+\beta} \in \operatorname{Tr}_{X}(\mathcal{H}) .
$$


Furthermore,

$$
\begin{aligned}
& \operatorname{det}_{\mathrm{X}}\left[\frac{\alpha\left[(A+\gamma I)(B+\mu I)^{-1}\right]^{p}+\beta\left[(A+\gamma I)(B+\mu I)^{-1}\right]^{-q}}{\alpha+\beta}\right] \\
&=\operatorname{det}_{\mathrm{X}}\left[\frac{\alpha\left(\Lambda+\frac{\gamma}{\mu} I\right)^{p}+\beta\left(\Lambda+\frac{\gamma}{\mu} I\right)^{-q}}{\alpha+\beta}\right] .
\end{aligned}
$$

Motivated by Eq. (52) and Propositions 2 and 3, the following is our definition of the Alpha-Beta Log-Determinant divergences on $\mathscr{P} \mathscr{C}_{2}(\mathcal{H})$.

Definition 2 (Alpha-Beta Log-Determinant divergences between positive definite Hilbert-Schmidt operators). Assume that $\operatorname{dim}(\mathcal{H})=\infty$. Let $\alpha>0, \beta>0$ be fixed. Let $r \in \mathbb{R}, r \neq 0$ be fixed. For $(A+\gamma I),(B+\mu I) \in \mathscr{P} \mathscr{C}_{2}(\mathcal{H})$, the $(\alpha, \beta)$-Log-Det divergence $D_{r}^{(\alpha, \beta)}[(A+\gamma I),(B+\mu I)]$ is defined to be

$$
\begin{aligned}
& D_{r}^{(\alpha, \beta)}[(A+\gamma I),(B+\mu I)] \\
& =\frac{1}{\alpha \beta} \log \left[\left(\frac{\gamma}{\mu}\right)^{r\left(\delta-\frac{\alpha}{\alpha+\beta}\right)} \operatorname{det}_{\mathrm{X}}\left(\frac{\alpha\left(\Lambda+\frac{\gamma}{\mu} I\right)^{r(1-\delta)}+\beta\left(\Lambda+\frac{\gamma}{\mu} I\right)^{-r \delta}}{\alpha+\beta}\right)\right]
\end{aligned}
$$

where $\Lambda+\frac{\gamma}{\mu} I=(B+\mu I)^{-1 / 2}(A+\gamma I)(B+\mu I)^{-1 / 2}, \delta=\frac{\alpha \gamma^{r}}{\alpha \gamma^{r}+\beta \mu^{r}}$. Equivalently,

$$
\begin{aligned}
& D_{r}^{(\alpha, \beta)}[(A+\gamma I),(B+\mu I)] \\
& =\frac{1}{\alpha \beta} \log \left[\left(\frac{\gamma}{\mu}\right)^{r\left(\delta-\frac{\alpha}{\alpha+\beta}\right)} \operatorname{det}_{\mathrm{X}}\left(\frac{\alpha\left(Z+\frac{\gamma}{\mu} I\right)^{r(1-\delta)}+\beta\left(Z+\frac{\gamma}{\mu} I\right)^{-r \delta}}{\alpha+\beta}\right)\right]
\end{aligned}
$$

where $Z+\frac{\gamma}{\mu} I=(A+\gamma I)(B+\mu I)^{-1}$.

While Definition 2 is stated using the extended Fredholm determinant $\operatorname{det}_{\mathrm{X}}$, the limiting cases $(\alpha>0, \beta=0)$ and $(\alpha=0, \beta>0)$ both require the concept of the extended Hilbert-Carleman determinant $\operatorname{det}_{2 \mathrm{X}}$.

Theorem 1 (Limiting case $\alpha>0, \beta \rightarrow 0)$. Let $\alpha>0$ be fixed. Assume that $r=r(\beta)$ is smooth, with $r(0)=r(\beta=0)$. Then

$$
\begin{aligned}
\lim _{\beta \rightarrow 0} D_{r}^{(\alpha, \beta)}[(A+\gamma I),(B+\mu I)] & =\frac{1}{\alpha^{2}}\left[\left(\frac{\mu}{\gamma}\right)^{r(0)}-1\right]\left(1+r(0) \log \frac{\mu}{\gamma}\right) \\
& -\frac{1}{\alpha^{2}}\left(\frac{\mu}{\gamma}\right)^{r(0)} \log \operatorname{det}_{2 \mathrm{X}}\left(\left[(A+\gamma I)^{-1}(B+\mu I)\right]^{r(0)}\right) .
\end{aligned}
$$


Theorem 2 (Limiting case $\alpha \rightarrow 0, \beta>0)$. Let $\beta>0$ be fixed. Assume that $r=r(\alpha)$ is smooth, with $r(0)=r(\alpha=0)$. Then

$$
\begin{aligned}
\lim _{\alpha \rightarrow 0} D_{r}^{(\alpha, \beta)}[(A+\gamma I),(B+\mu I)] & =\frac{1}{\beta^{2}}\left[\left(\frac{\gamma}{\mu}\right)^{r(0)}-1\right]\left(1+r(0) \log \frac{\gamma}{\mu}\right) \\
& -\frac{1}{\beta^{2}}\left(\frac{\gamma}{\mu}\right)^{r(0)} \log \operatorname{det}_{2 \mathrm{X}}\left(\left[(B+\mu I)^{-1}(A+\gamma I)\right]^{r(0)}\right) .
\end{aligned}
$$

Motivated by Theorems 1 and 2 , the following is our definition of $D_{r}^{(\alpha, 0)}[(A+$ $\gamma I),(B+\mu I)]$ and $D_{r}^{(0, \beta)}[(A+\gamma I),(B+\mu I)], \alpha>0, \beta>0$.

Definition 3 (Limiting cases). Let $\alpha, \beta>0$ be fixed. Let $r \in \mathbb{R}, r \neq 0$ be fixed. For $(A+\gamma I),(B+\mu I) \in \mathscr{P} \mathscr{C}_{2}(\mathcal{H})$, the divergence $D_{r}^{(\alpha, 0)}[(A+\gamma I),(B+\mu I)]$ is defined to be

$$
\begin{aligned}
D_{r}^{(\alpha, 0)}[(A+\gamma I),(B+\mu I)] & =\frac{1}{\alpha^{2}}\left[\left(\frac{\mu}{\gamma}\right)^{r}-1\right]\left(1+r \log \frac{\mu}{\gamma}\right) \\
& -\frac{1}{\alpha^{2}}\left(\frac{\mu}{\gamma}\right)^{r} \log \operatorname{det}_{2 \mathrm{X}}\left(\left[(A+\gamma I)^{-1}(B+\mu I)\right]^{r}\right)
\end{aligned}
$$

Similarly, the divergence $D_{r}^{(0, \beta)}[(A+\gamma I),(B+\mu I)]$ is defined to be

$$
\begin{aligned}
D_{r}^{(0, \beta)}[(A+\gamma I),(B+\mu I)] & =\frac{1}{\beta^{2}}\left[\left(\frac{\gamma}{\mu}\right)^{r}-1\right]\left(1+r \log \frac{\gamma}{\mu}\right) \\
& -\frac{1}{\beta^{2}}\left(\frac{\gamma}{\mu}\right)^{r} \log \operatorname{det}_{2 \mathrm{X}}\left(\left[(B+\mu I)^{-1}(A+\gamma I)\right]^{r}\right)
\end{aligned}
$$

For the case $(A+\gamma I),(B+\mu I) \in \mathscr{P} \mathscr{C}_{1}(\mathcal{H})$, from Definition 3, we recover the formulation stated in Definition 2 in [2], as follows.

Corollary 2. Let $\alpha, \beta>0$ be fixed. Let $r \in \mathbb{R}, r \neq 0$ be fixed. Assume that $(A+$ $\gamma I),(B+\mu I) \in \mathscr{P} \mathscr{C}_{1}(\mathcal{H})$. Then in Definition 3,

$$
\begin{aligned}
D_{r}^{(\alpha, 0)}[(A+\gamma I),(B+\mu I)] & =\frac{r}{\alpha^{2}}\left[\left(\frac{\mu}{\gamma}\right)^{r}-1\right] \log \frac{\mu}{\gamma} \\
& -\frac{1}{\alpha^{2}}\left(\frac{\mu}{\gamma}\right)^{r} \log \operatorname{det}_{\mathrm{X}}\left[(A+\gamma I)^{-1}(B+\mu I)\right]^{r} \\
& +\frac{1}{\alpha^{2}} \operatorname{tr}_{X}\left(\left[(A+\gamma I)^{-1}(B+\mu I)\right]^{r}-I\right)
\end{aligned}
$$




$$
\begin{aligned}
D_{r}^{(0, \beta)}[(A+\gamma I),(B+\mu I)] & =\frac{r}{\beta^{2}}\left[\left(\frac{\gamma}{\mu}\right)^{r}-1\right] \log \frac{\gamma}{\mu} \\
& -\frac{1}{\beta^{2}}\left(\frac{\gamma}{\mu}\right)^{r} \log \operatorname{det}_{\mathrm{X}}\left[(B+\mu I)^{-1}(A+\gamma I)\right]^{r} \\
& +\frac{1}{\beta^{2}} \operatorname{tr}_{X}\left(\left[(B+\mu I)^{-1}(A+\gamma I)\right]^{r}-I\right)
\end{aligned}
$$

The following is the generalization of Theorem 9 in [2] to positive definite HilbertSchmidt operators.

Theorem 3 (Limiting case $(0,0))$. Assume that $(A+\gamma I),(B+\mu I) \in \mathscr{P} \mathscr{C}_{2}(\mathcal{H})$. Assume that $r=r(\alpha)$ is smooth, with $r(0)=0, r^{\prime}(0) \neq 0$, and $r(\alpha) \neq 0$ for $\alpha \neq 0$. Then

$$
\lim _{\alpha \rightarrow 0} D_{r}^{(\alpha, \alpha)}[(A+\gamma I),(B+\mu I)]=\frac{\left[r^{\prime}(0)\right]^{2}}{8} d_{\mathrm{aiHS}}^{2}[(A+\gamma I),(B+\mu I)] .
$$

In particular, for $r=2 \alpha$,

$$
\lim _{\alpha \rightarrow 0} D_{2 \alpha}^{(\alpha, \alpha)}[(A+\gamma I),(B+\mu I)]=\frac{1}{2} d_{\mathrm{aiHS}}^{2}[(A+\gamma I),(B+\mu I)] .
$$

The following is the generalization of Theorem 3 in [2] to positive definite HilbertSchmidt operators.

Theorem 4 (Symmetric divergences). The parametrized family $D_{2 \alpha}^{(\alpha, \alpha)}[(A+\gamma I),(B+$ $\mu I)], \alpha \geq 0$, is a family of symmetric divergences on $\mathscr{P} \mathscr{C}_{2}(\mathcal{H})$, with $\alpha=0$ corresponding to the infinite-dimensional affine-invariant Riemannian distance above and $\alpha=1 / 2$ corresponding to the infinite-dimensional symmetric Stein divergence, which is given by $\frac{1}{4} d_{\log \operatorname{det}}^{0}[(A+\gamma I),(B+\mu I)]$.

\section{Properties of the Log-Determinant divergences}

The following results establish several important properties of $D_{r}^{(\alpha, \beta)}$ as defined above, which generalize those from both the finite-dimensional setting $[9,3]$ and the infinite-dimensional Alpha Log-Det divergences [1] and Alpha-Beta Log-Det divergences [2] for positive definite trace class operators.

In the following theorems, $(A+\gamma I),(B+\mu I) \in \mathscr{P} \mathscr{C}_{2}(\mathcal{H})$. 


\section{Theorem 5 (Positivity).}

$$
\begin{aligned}
& D_{r}^{(\alpha, \beta)}[(A+\gamma I),(B+\mu I)] \geq 0, \\
& D_{r}^{(\alpha, \beta)}[(A+\gamma I),(B+\mu I)]=0 \Longleftrightarrow A=B, \gamma=\mu .
\end{aligned}
$$

Theorem 6 (Dual symmetry).

$$
D_{r}^{(\beta, \alpha)}[(B+\mu I),(A+\gamma I)]=D_{r}^{(\alpha, \beta)}[(A+\gamma I),(B+\mu I)] .
$$

In particular, for $\beta=\alpha$, we have

$$
D_{r}^{(\alpha, \alpha)}[(B+\mu I),(A+\gamma I)]=D_{r}^{(\alpha, \alpha)}[(A+\gamma I),(B+\mu I)]
$$

Theorem 7 (Dual invariance under inversion).

$$
D_{r}^{(\alpha, \beta)}\left[(A+\gamma I)^{-1},(B+\mu I)^{-1}\right]=D_{-r}^{(\alpha, \beta)}[(A+\gamma I),(B+\mu I)]
$$

Theorem 8 (Affine invariance). For any $(A+\gamma I),(B+\mu I) \in \mathscr{P} \mathscr{C}_{2}(\mathcal{H})$ and any invertible $(C+\nu I) \in \operatorname{HS}_{X}(\mathcal{H}), \nu \neq 0$,

$$
\begin{aligned}
& D_{r}^{(\alpha, \beta)}\left[(C+\nu I)(A+\gamma I)(C+\nu I)^{*},(C+\nu I)(B+\mu I)(C+\nu I)^{*}\right] \\
& =D_{r}^{(\alpha, \beta)}[(A+\gamma I),(B+\mu I)] .
\end{aligned}
$$

Theorem 9 (Invariance under unitary transformations). For any $(A+\gamma I),(B+$ $\mu I) \in \mathscr{P} \mathscr{C}_{2}(\mathcal{H})$ and any $C \in \mathcal{L}(\mathcal{H})$, with $C C^{*}=C^{*} C=I$,

$$
D_{r}^{(\alpha, \beta)}\left[C(A+\gamma I) C^{*}, C(B+\mu I) C^{*}\right]=D_{r}^{(\alpha, \beta)}[(A+\gamma I),(B+\mu I)] .
$$

\section{Proofs of main results}

\subsection{Proofs of the properties of the extended Hilbert-Carleman determinant}

Proof of Lemma 1. By definition of the Hilbert-Carleman determinant and the assumption that $I+A>0$, we have

$$
\operatorname{det}_{2}(I+A)=\operatorname{det}[(I+A) \exp (-A)]=\prod_{k=1}^{\infty}\left[\left(1+\lambda_{k}\right) \exp \left(-\lambda_{k}\right)\right]>0
$$


Thus $\log \operatorname{det}_{2}(I+A)$ is well-defined and finite, and is given by the series

$$
\log \operatorname{det}_{2}(I+A)=\sum_{k=1}^{\infty}\left[\log \left(1+\lambda_{k}\right)-\lambda_{k}\right]
$$

which necessarily has a finite value.

For the second statement, consider the function $f(x)=\log (1+x)-x$ for $x>-1$. We have $f^{\prime}(x)=-\frac{x}{1+x}$, with $f^{\prime}(x)>0$ for $-1<x<0$ and $f^{\prime}(x)<0$ for $x>0$. Thus $f$ has a unique global maximum $f_{\max }=f(0)=0$. Thus $\forall k \in \mathbb{N}$,

$$
\log \left(1+\lambda_{k}\right)-\lambda_{k} \leq 0, \quad \text { with equality if and only } \lambda_{k}=0
$$

It then follows that $\log (I+A) \leq 0$, with equality if and only $\lambda_{k}=0 \forall k \in \mathbb{N}$, that is if and only if $A=0$.

Proof of Lemma 2. We make use of the result that $R_{2}(A)=(I+A) \exp (-A)-I \in$ $\operatorname{Tr}(\mathcal{H})$ for $A \in \operatorname{HS}(\mathcal{H})$. Thus

$R_{2, \gamma}(A)=(A+\gamma I) \exp (-A / \gamma)-\gamma I=\gamma\left[\left(\frac{A}{\gamma}+I\right) \exp (-A / \gamma)-I\right] \in \operatorname{Tr}(\mathcal{H})$

and hence $R_{2, \gamma}(A)+\gamma I=(A+\gamma I) \exp (-A / \gamma) \in \operatorname{Tr}_{X}(\mathcal{H})$. Since $R_{2, \gamma}(A) \in \operatorname{Tr}(\mathcal{H})$, the infinite product

$$
\prod_{k=1}^{\infty}\left[\left(\lambda_{k}+\gamma\right) \exp \left(-\lambda_{k} / \gamma\right)-\gamma+1\right]=\operatorname{det}\left[R_{2, \gamma}(A)+I\right]
$$

converges to a finite value.

Proof of Lemma 3 (Factorization Rule). We have $\left(\frac{A}{\gamma}+I\right) \exp (-A / \gamma)-I \in \operatorname{Tr}(\mathcal{H})$ and thus for the operator

$$
(A+\gamma I) \exp (-A / \gamma)=\gamma\left[\left(\frac{A}{\gamma}+I\right) \exp (-A / \gamma)-I\right]+\gamma I \in \operatorname{Tr}_{X}(\mathcal{H})
$$

its extended Fredholm determinant is given by

$$
\operatorname{det}_{\mathrm{X}}[(A+\gamma I) \exp (-A / \gamma)]=\gamma \operatorname{det}\left[\left(\frac{A}{\gamma}+I\right) \exp (-A / \gamma)\right]=\gamma \operatorname{det}_{2}\left(\frac{A}{\gamma}+I\right) .
$$

This completes the proof. 
Proof of Lemma 4 (Similarity Invariant). Since $\operatorname{HS}(\mathcal{H})$ is a two-sided ideal in $\mathcal{L}(\mathcal{H})$, we have $C A C^{-1} \in \operatorname{HS}(\mathcal{H})$. Thus

$$
C(A+\gamma I) C^{-1}=C A C^{-1}+\gamma I \in \operatorname{HS}_{X}(\mathcal{H}) .
$$

By definition of the extended Hilbert-Carleman determinant, we have

$$
\begin{aligned}
& \operatorname{det}_{2 \mathrm{X}}\left[C(A+\gamma I) C^{-1}\right]=\operatorname{det}_{\mathrm{X}}\left[C(A+\gamma I) C^{-1} \exp \left(-C A C^{-1} / \gamma\right)\right] \\
& =\operatorname{det}_{\mathrm{X}}\left[C(A+\gamma I) C^{-1}\left(C \exp (-A / \gamma) C^{-1}\right)\right]=\operatorname{det}_{\mathrm{X}}\left[C(A+\gamma I) \exp (-A / \gamma) C^{-1}\right] \\
& =\operatorname{det}_{\mathrm{X}}[(A+\gamma I) \exp (-A / \gamma) \quad \text { by Eq. (34) } \\
& =\operatorname{det}_{2 \mathrm{X}}(A+\gamma I) .
\end{aligned}
$$

This completes the proof.

Proof of Lemma 5 (Commutativity). Consider the first assumption, that is $(A+\gamma I),(B+$ $\mu I) \in \mathscr{P} \mathscr{C}_{2}(\mathcal{H})$. We write $(A+\gamma I)(B+\mu I)$ and $(B+\mu I)(A+\gamma I)$ as

$$
\begin{aligned}
& (A+\gamma I)(B+\mu I)=(A+\gamma I)^{1 / 2}\left[(A+\gamma I)^{1 / 2}(B+\mu I)(A+\gamma I)^{1 / 2}\right](A+\gamma I)^{-1 / 2}, \\
& (B+\mu I)(A+\gamma I)=(A+\gamma I)^{-1 / 2}\left[(A+\gamma I)^{1 / 2}(B+\mu I)(A+\gamma I)^{1 / 2}\right](A+\gamma I)^{1 / 2} .
\end{aligned}
$$

By Lemma 4, we then have

$$
\begin{aligned}
\operatorname{det}_{2 \mathrm{X}}[(A+\gamma I)(B+\mu I)] & =\operatorname{det}_{2 \mathrm{X}}\left[(A+\gamma I)^{1 / 2}(B+\mu I)(A+\gamma I)^{1 / 2}\right] \\
& =\operatorname{det}_{2 \mathrm{X}}[(B+\mu I)(A+\gamma I)] .
\end{aligned}
$$

The third statement is proved similarly.

Under the second assumption, that is $(A+\gamma I),(B+\mu I) \in \operatorname{Tr}_{X}(\mathcal{H}), \gamma \neq 0, \mu \neq 0$, we have by definition

$$
\begin{aligned}
& \operatorname{det}_{2 \mathrm{X}}[(A+\gamma I)(B+\mu I)]=\operatorname{det}_{\mathrm{X}}[(A+\gamma I)(B+\mu I)] \exp \left(-\frac{\operatorname{tr}[\mu A+\gamma B+A B]}{\gamma \mu}\right) \\
& =\operatorname{det}_{\mathrm{X}}[(B+\mu I)(A+\gamma I)] \exp \left(-\frac{\operatorname{tr}[\mu A+\gamma B+B A]}{\gamma \mu}\right) \\
& =\operatorname{det}_{2 \mathrm{X}}[(B+\mu I)(A+\gamma I)] .
\end{aligned}
$$

Here we have made use of the properties $\operatorname{det}_{\mathrm{X}}[(A+\gamma I)(B+\mu I)]=\operatorname{det}_{\mathrm{X}}(A+$ $\gamma I) \operatorname{det}_{\mathrm{X}}(B+\mu I)=\operatorname{det}_{\mathrm{X}}[(B+\mu I)(A+\gamma I)]$ and the commutativity of the trace, namely $\operatorname{tr}(A B)=\operatorname{tr}(B A)$. This completes the proof. 
Proof of Lemma 6. We rewrite $(A+\gamma I)(B+\mu I)^{-1}$ as

$$
\begin{aligned}
& (A+\gamma I)(B+\mu I)^{-1} \\
& =(B+\mu I)^{1 / 2}\left[(B+\mu I)^{-1 / 2}(A+\gamma I)(B+\mu I)^{-1 / 2}\right](B+\mu I)^{-1 / 2} \\
& =(B+\mu I)^{1 / 2}(\Lambda+\nu I)(B+\mu I)^{-1 / 2} .
\end{aligned}
$$

Similarly,

$$
(B+\mu I)^{-1}(A+\gamma I)=(B+\mu I)^{-1 / 2}(\Lambda+\nu I)(B+\mu I)^{1 / 2} .
$$

By definition of the power function, we then have for any $\alpha \in \mathbb{R}$

$$
\begin{aligned}
& {\left[(A+\gamma I)(B+\mu I)^{-1}\right]^{\alpha}=(B+\mu I)^{1 / 2}(\Lambda+\nu I)^{\alpha}(B+\mu I)^{-1 / 2},} \\
& {\left[(B+\mu I)^{-1}(A+\gamma I)\right]^{\alpha}=(B+\mu I)^{-1 / 2}(\Lambda+\nu I)^{\alpha}(B+\mu I)^{1 / 2} .}
\end{aligned}
$$

Thus by Lemma 4, we obtain

$$
\begin{aligned}
\operatorname{det}_{2 \mathrm{X}}\left(\left[(A+\gamma I)(B+\mu I)^{-1}\right]^{\alpha}\right) & =\operatorname{det}_{2 \mathrm{X}}\left[(\Lambda+\nu I)^{\alpha}\right] \\
& =\operatorname{det}_{2 \mathrm{X}}\left(\left[(B+\mu I)^{-1}(A+\gamma I)\right]^{\alpha}\right) .
\end{aligned}
$$

This completes the proof.

Lemma 9. Let $r \neq 0$ be fixed. The function $f(x)=x^{r}-1-r \log (x)$ for $x>0$ has a unique global minimum $f_{\min }=f(1)=0$. In other words, $f(x) \geq 0 \forall x>0$, with equality if and only if $x=1$.

Proof of Lemma 9. We have $f^{\prime}(x)=\frac{r\left(x^{r}-1\right)}{x}$. When $r>0$, we have $x^{r}<1$ for $0<x<1$ and $x^{r}>1$ for $x>1$. When $r<0$, we have $x^{r}>1$ for $0<x<1$ and $x^{r}<1$ for $x>1$. Thus, for all $r \neq 0$, we have $f^{\prime}(x)<0$ when $0<x<1$ and $f^{\prime}(x)>0$ when $x>1$. Hence $f$ has a unique global minimum $f_{\min }=f(1)=0$.

Proof of Lemma 7. By Proposition 2 in [15], we have $\log (I+A) \in \mathrm{HS}(\mathcal{H})$ for $(I+$ $A) \in \mathscr{P} \mathscr{C}_{2}(\mathcal{H})$. By definition of the power function, we have

$$
(I+A)^{\alpha}=\exp [\alpha \log (I+A)]=I+\sum_{j=1}^{\infty} \frac{\alpha^{j}}{j !}[\log (I+A)]^{j} .
$$


Since $\operatorname{HS}(\mathcal{H})$ is a Banach algebra under the Hilbert-Schmidt norm, we then have

$$
\begin{aligned}
\left\|(I+A)^{\alpha}-I\right\|_{\mathrm{HS}} & =\left\|\sum_{j=1}^{\infty} \frac{\alpha^{j}}{j !}[\log (I+A)]^{j}\right\|_{\mathrm{HS}} \leq \sum_{j=1}^{\infty} \frac{|\alpha|^{j}}{j !}\|\log (I+A)\|_{\mathrm{HS}}^{j} \\
& =\exp \left(|\alpha|\|\log (I+A)\|_{\mathrm{HS}}\right)-1<\infty .
\end{aligned}
$$

Thus $(I+A)^{\alpha}-I \in \operatorname{HS}(\mathcal{H})$. By definition of the Hilbert-Carleman determinant, we then have

$$
\begin{aligned}
\operatorname{det}_{2}\left[(I+A)^{\alpha}\right] & =\operatorname{det}\left[(I+A)^{\alpha} \exp \left(-\left[(I+A)^{\alpha}-I\right]\right)\right] \\
& =\prod_{k=1}^{\infty}\left(1+\lambda_{k}\right)^{\alpha} \exp \left[1-\left(1+\lambda_{k}\right)^{\alpha}\right]<\infty
\end{aligned}
$$

Thus the following quantity is well-defined and finite

$$
\log \operatorname{det}_{2}\left[(I+A)^{\alpha}\right]=\sum_{k=1}^{\infty}\left[\alpha \log \left(1+\lambda_{k}\right)+1-\left(1+\lambda_{k}\right)^{\alpha}\right] .
$$

The statements for the case $I+A \in \mathscr{P} \mathscr{C}_{1}(\mathcal{H})$ are then obvious from the above series expansions.

By Lemma 9, we have $\forall k \in \mathbb{N}$,

$$
\alpha \log \left(1+\lambda_{k}\right)+1-\left(1+\lambda_{k}\right)^{\alpha} \leq 0
$$

with equality if and only if $\lambda_{k}=0$. Thus it follows that

$$
\log \operatorname{det}_{2}\left[(I+A)^{\alpha}\right] \leq 0
$$

with equality if and only if $\lambda_{k}=0 \forall k \in \mathbb{N}$, that is if and only if $A=0$ (by the assumption that $I+A>0)$.

Proof of Lemma 8. By definition of the power function, we have

$$
\begin{aligned}
(A+\gamma I)^{\alpha} & =\exp [\alpha \log (A+\gamma I)]=\exp \left[(\alpha \log \gamma) I+\alpha \log \left(\frac{A}{\gamma}+I\right)\right] \\
& =\gamma^{\alpha}\left(\frac{A}{\gamma}+I\right)^{\alpha}=\gamma^{\alpha}\left[\left(\frac{A}{\gamma}+I\right)^{\alpha}-I\right]+\gamma^{\alpha} I
\end{aligned}
$$

where $\left[\left(\frac{A}{\gamma}+I\right)^{\alpha}-I\right] \in \operatorname{Sym}(\mathcal{H}) \cap \operatorname{HS}(\mathcal{H})$ by Lemma 7 . Thus it follows that $(A+\gamma I)^{\alpha}-\gamma^{\alpha} I \in \operatorname{Sym}(\mathcal{H}) \cap \operatorname{HS}(\mathcal{H})$. Therefore, the extended Hilbert-Carleman 
determinant of $(A+\gamma I)^{\alpha}$ is well-defined and finite. By the Factorization Rule (Lemma 3) and Lemma 7, we have

$$
\begin{aligned}
\operatorname{det}_{2 \mathrm{X}}\left[(A+\gamma I)^{\alpha}\right] & =\gamma^{\alpha} \operatorname{det}_{2}\left[\left(\frac{A}{\gamma}+I\right)^{\alpha}\right] \\
& =\gamma^{\alpha} \prod_{k=1}^{\infty}\left(1+\frac{\lambda_{k}}{\gamma}\right)^{\alpha} \exp \left[1-\left(1+\frac{\lambda_{k}}{\gamma}\right)^{\alpha}\right]<\infty
\end{aligned}
$$

Consequently, the following quantity is also finite

$$
\log \operatorname{det}_{2 \mathrm{X}}\left[(A+\gamma I)^{\alpha}\right]=\alpha \log \gamma+\sum_{k=1}^{\infty}\left[\alpha \log \left(1+\frac{\lambda_{k}}{\gamma}\right)+1-\left(1+\frac{\lambda_{k}}{\gamma}\right)^{\alpha}\right] .
$$

If $A+\gamma I \in \mathscr{P} \mathscr{C}_{1}(\mathcal{H})$, then $\left(\frac{A}{\gamma}+I\right)^{\alpha}-I \in \operatorname{Tr}(\mathcal{H})$ (see Lemma 6 in [2], or by using a similar argument as in Lemma 7). Thus the following infinite product and series

$$
\begin{gathered}
\operatorname{det}\left(\frac{A}{\gamma}+I\right)^{\alpha}=\prod_{k=1}^{\infty}\left(\frac{\lambda_{k}}{\gamma}+1\right)^{\alpha}, \\
\alpha \log \operatorname{det}\left(\frac{A}{\gamma}+I\right)=\alpha \sum_{k=1}^{\infty} \log \left(\frac{\lambda_{k}}{\gamma}+1\right), \\
\operatorname{tr}\left[\left(\frac{A}{\gamma}+I\right)^{\alpha}-I\right]=\sum_{k=1}^{\infty}\left[\left(1+\frac{\lambda_{k}}{\gamma}\right)^{\alpha}-1\right]
\end{gathered}
$$

converge to finite values. These give the last statements of the lemma.

\subsection{Proofs for the definition of the Log-Determinant divergences}

Lemma 10. Let $\alpha>0, \beta>0, \gamma>0$ be fixed. Let $p, q \in \mathbb{R}$ be such that $p \alpha \gamma^{p}=$ $q \beta \gamma^{-q}$. Then

$$
\lim _{x \rightarrow 0} \frac{1-\frac{\alpha \gamma^{p}(1+x)^{p}+\beta \gamma^{-q}(1+x)^{-q}}{\alpha \gamma^{p}+\beta \gamma^{-q}}}{x^{2}}=-\frac{p(p-1) \alpha \gamma^{p}+q(q+1) \beta \gamma^{-q}}{2\left(\alpha \gamma^{p}+\beta \gamma^{-q}\right)} .
$$

Proof of Lemma 10. Since the limit has the form $\frac{0}{0}$, by L'Hopital's rule, we have

$$
\begin{aligned}
& \lim _{x \rightarrow 0} \frac{1-\frac{\alpha \gamma^{p}(1+x)^{p}+\beta \gamma^{-q}(1+x)^{-q}}{\alpha \gamma^{p}+\beta \gamma^{-q}}}{x^{2}} \\
& =-\frac{1}{2\left(\alpha \gamma^{p}+\beta \gamma^{-q}\right)} \lim _{x \rightarrow 0} \frac{p \alpha \gamma^{p}(1+x)^{p-1}-q \beta \gamma^{-q}(1+x)^{-q-1}}{x} .
\end{aligned}
$$


By assumption, we have $p \alpha \gamma^{p}=q \beta \gamma^{-q}$, so that the previous limit also has the form $\frac{0}{0}$. Applying L'Hopital's rule one more time, we obtain

$$
\begin{aligned}
& -\frac{1}{2\left(\alpha \gamma^{p}+\beta \gamma^{-q}\right)} \lim _{x \rightarrow 0}\left[p(p-1) \alpha \gamma^{p}(1+x)^{p-2}+q(q+1) \beta \gamma^{-q}(1+x)^{-q-2}\right] \\
& =-\frac{p(p-1) \alpha \gamma^{p}+q(q+1) \beta \gamma^{-q}}{2\left(\alpha \gamma^{p}+\beta \gamma^{-q}\right)} .
\end{aligned}
$$

This completes the proof.

Corollary 3. Let $A \in \operatorname{Sym}(\mathcal{H}) \cap \operatorname{HS}(\mathcal{H})$ be such that $I+A>0$. Let $\alpha>0, \beta>$ $0, \gamma>0$ be fixed. Let $p, q \in \mathbb{R}$ be such that $p \alpha \gamma^{p}=q \beta \gamma^{-q}$. Then

$$
I-\frac{\alpha \gamma^{p}(I+A)^{p}+\beta \gamma^{-q}(I+A)^{-q}}{\alpha \gamma^{p}+\beta \gamma^{-q}} \in \operatorname{Sym}(\mathcal{H}) \cap \operatorname{Tr}(\mathcal{H}) .
$$

Proof of Corollary 3. Let $\left\{\lambda_{k}\right\}_{k=1}^{\infty}$ denote the eigenvalues of $A$ then $\lim _{k \rightarrow \infty} \lambda_{k}=0$.

By Lemma 10, we have

$$
\lim _{k \rightarrow \infty} \frac{1-\frac{\alpha \gamma^{p}\left(1+\lambda_{k}\right)^{p}+\beta \gamma^{-q}\left(1+\lambda_{k}\right)^{-q}}{\alpha \gamma^{p}+\beta \gamma^{-q}}}{\lambda_{k}^{2}}=-\frac{p(p-1) \alpha \gamma^{p}+q(q+1) \beta \gamma^{-q}}{2\left(\alpha \gamma^{p}+\beta \gamma^{-q}\right)} .
$$

This implies that there exists a constant $C>0$, independent of $k$, and a number $N=$ $N(C) \in \mathbb{N}$, such that

$$
\left|1-\frac{\alpha \gamma^{p}\left(1+\lambda_{k}\right)^{p}+\beta \gamma^{-q}\left(1+\lambda_{k}\right)^{-q}}{\alpha \gamma^{p}+\beta \gamma^{-q}}\right| \leq C \lambda_{k}^{2} \quad \forall k \geq N .
$$

Since $\sum_{k=1}^{\infty} \lambda_{k}^{2}<\infty$ by assumption, it then follows that

$$
\sum_{k=1}^{\infty}\left|1-\frac{\alpha \gamma^{p}\left(1+\lambda_{k}\right)^{p}+\beta \gamma^{-q}\left(1+\lambda_{k}\right)^{-q}}{\alpha \gamma^{p}+\beta \gamma^{-q}}\right|<\infty
$$

which gives us the desired result.

Proof of Proposition 2. Since $(A+\gamma I),(B+\mu I) \in \mathscr{P} \mathscr{C}_{2}(\mathcal{H})$, we have $\Lambda+\frac{\gamma}{\mu} I=$ $(B+\mu I)^{-1 / 2}(A+\gamma I)(B+\mu I)^{-1 / 2} \in \mathscr{P} \mathscr{C}_{2}(\mathcal{H})$, with $\Lambda \in \operatorname{HS}(\mathcal{H})$. Thus it is obvious that $\frac{\alpha\left(\Lambda+\frac{\gamma}{\mu} I\right)^{p}+\beta\left(\Lambda+\frac{\gamma}{\mu} I\right)^{-q}}{\alpha+\beta}$ is also positive definite. Let us show that it in 
extended trace class operator. Consider the expansion

$$
\begin{aligned}
& \frac{\alpha\left(\Lambda+\frac{\gamma}{\mu} I\right)^{p}+\beta\left(\Lambda+\frac{\gamma}{\mu} I\right)^{-q}}{\alpha+\beta} \\
& =\frac{\alpha\left(\frac{\gamma}{\mu}\right)^{p}+\beta\left(\frac{\gamma}{\mu}\right)^{-q}}{\alpha+\beta} \frac{\alpha\left(\frac{\gamma}{\mu}\right)^{p}\left(\frac{\mu}{\gamma} \Lambda+I\right)^{p}+\beta\left(\frac{\gamma}{\mu}\right)^{-q}\left(\frac{\mu}{\gamma} \Lambda+I\right)^{-q}}{\alpha\left(\frac{\gamma}{\mu}\right)^{p}+\beta\left(\frac{\gamma}{\mu}\right)^{-q}} \\
& =\frac{\alpha\left(\frac{\gamma}{\mu}\right)^{p}+\beta\left(\frac{\gamma}{\mu}\right)^{-q}}{\alpha+\beta}\left[I-\left(I-\frac{\alpha\left(\frac{\gamma}{\mu}\right)^{p}\left(\frac{\mu}{\gamma} \Lambda+I\right)^{p}+\beta\left(\frac{\gamma}{\mu}\right)^{-q}\left(\frac{\mu}{\gamma} \Lambda+I\right)^{-q}}{\alpha\left(\frac{\gamma}{\mu}\right)^{p}+\beta\left(\frac{\gamma}{\mu}\right)^{-q}}\right)\right] .
\end{aligned}
$$

By Corollary 3, we have $\left(I-\frac{\alpha\left(\frac{\gamma}{\mu}\right)^{p}\left(\frac{\mu}{\gamma} \Lambda+I\right)^{p}+\beta\left(\frac{\gamma}{\mu}\right)^{-q}\left(\frac{\mu}{\gamma} \Lambda+I\right)^{-q}}{\alpha\left(\frac{\gamma}{\mu}\right)^{p}+\beta\left(\frac{\gamma}{\mu}\right)^{-q}}\right) \in \operatorname{Sym}(\mathcal{H}) \cap \operatorname{Tr}(\mathcal{H})$.

Thus it follows that $\frac{\alpha\left(\Lambda+\frac{\gamma}{\mu} I\right)^{p}+\beta\left(\Lambda+\frac{\gamma}{\mu} I\right)^{-q}}{\alpha+\beta} \in \operatorname{Tr}_{X}(\mathcal{H})$. This completes the proof.

Proof of Proposition 3. By Proposition 2, we have

$$
\frac{\alpha\left(\Lambda+\frac{\gamma}{\mu} I\right)^{p}+\beta\left(\Lambda+\frac{\gamma}{\mu} I\right)^{-q}}{\alpha+\beta} \in \mathscr{P} \mathscr{C}_{1}(\mathcal{H}) .
$$

Thus its extended Fredholm determinant $\operatorname{det}_{\mathrm{X}}$ is well-defined and finite.

By Proposition 1 , we have for any $p \in \mathbb{R}$,

$$
\left[(A+\gamma I)(B+\mu I)^{-1}\right]^{p}=(B+\mu I)^{1 / 2}\left(\Lambda+\frac{\gamma}{\mu} I\right)^{p}(B+\mu I)^{-1 / 2} \in \operatorname{HS}_{X}(\mathcal{H}) .
$$

Thus it follows that

$$
\begin{aligned}
& \frac{\alpha\left[(A+\gamma I)(B+\mu I)^{-1}\right]^{p}+\beta\left[(A+\gamma I)(B+\mu I)^{-1}\right]^{-q}}{\alpha+\beta} \\
& =(B+\mu I)^{1 / 2}\left[\frac{\alpha\left(\Lambda+\frac{\gamma}{\mu} I\right)^{p}+\beta\left(\Lambda+\frac{\gamma}{\mu} I\right)^{-q}}{\alpha+\beta}\right](B+\mu I)^{-1 / 2} \in \operatorname{Tr}_{X}(\mathcal{H}) .
\end{aligned}
$$

Thus by Eq. (34), we obtain

$$
\begin{aligned}
& \operatorname{det}_{\mathrm{X}}\left[\frac{\alpha\left[(A+\gamma I)(B+\mu I)^{-1}\right]^{p}+\beta\left[(A+\gamma I)(B+\mu I)^{-1}\right]^{-q}}{\alpha+\beta}\right] \\
& =\operatorname{det}_{\mathrm{X}}\left[\frac{\alpha\left(\Lambda+\frac{\gamma}{\mu} I\right)^{p}+\beta\left(\Lambda+\frac{\gamma}{\mu} I\right)^{-q}}{\alpha+\beta}\right] .
\end{aligned}
$$

This completes the proof.

Proof of Theorems 1 and 2. Let $\left\{\lambda_{j}\right\}_{j=1}^{\infty}$ be the eigenvalues of $\Lambda$. By Theorem 8 in 
[2], we have the following expansion

$$
\begin{aligned}
D_{r}^{(\alpha, \beta)}[(A+\gamma I),(B+\mu I)] & =\frac{r\left(\delta-\frac{\alpha}{\alpha+\beta}\right)}{\alpha \beta} \log \left(\frac{\gamma}{\mu}\right)+\frac{1}{\alpha \beta} \log \left(\frac{\alpha\left(\frac{\gamma}{\mu}\right)^{p}+\beta\left(\frac{\gamma}{\mu}\right)^{-q}}{\alpha+\beta}\right) \\
& +\frac{1}{\alpha \beta} \log \operatorname{det}\left(\frac{\alpha\left(\Lambda+\frac{\gamma}{\mu} I\right)^{p}+\beta\left(\Lambda+\frac{\gamma}{\mu} I\right)^{-q}}{\alpha\left(\frac{\gamma}{\mu}\right)^{p}+\beta\left(\frac{\gamma}{\mu}\right)^{-q}}\right) \\
& =\frac{r\left(\delta-\frac{\alpha}{\alpha+\beta}\right)}{\alpha \beta} \log \left(\frac{\gamma}{\mu}\right)+\frac{1}{\alpha \beta} \log \left(\frac{\alpha\left(\frac{\gamma}{\mu}\right)^{p}+\beta\left(\frac{\gamma}{\mu}\right)^{-q}}{\alpha+\beta}\right) \\
& +\frac{1}{\alpha \beta} \sum_{j=1}^{\infty} \log \left(\frac{\alpha\left(\lambda_{j}+\frac{\gamma}{\mu}\right)^{p}+\beta\left(\lambda_{j}+\frac{\gamma}{\mu}\right)^{-q}}{\alpha\left(\frac{\gamma}{\mu}\right)^{p}+\beta\left(\frac{\gamma}{\mu}\right)^{-q}}\right),
\end{aligned}
$$

where $p=p(\beta)=r(1-\delta)=\frac{r \beta}{\alpha\left(\frac{\gamma}{\mu}\right)^{r}+\beta}, q=q(\beta)=r \delta=\frac{r \alpha\left(\frac{\gamma}{\mu}\right)^{r}}{\alpha\left(\frac{\gamma}{\mu}\right)^{r}+\beta}$.

Let $\nu=\frac{\gamma}{\mu}$. By the same argument as in the proof of Theorem 11 in [2], we have

$$
\begin{gathered}
\lim _{\beta \rightarrow 0} D_{r}^{(\alpha, \beta)}[(A+\gamma I),(B+\mu I)]=\frac{1}{\alpha^{2}}\left[\nu^{-r(0)}+r(0) \log (\nu)-1\right] \\
+\frac{1}{\alpha^{2}} \sum_{j=1}^{\infty}\left[\frac{r(0)}{\nu^{r(0)}} \log \left(\frac{\lambda_{j}}{\nu}+1\right)+\frac{1}{\left(\lambda_{j}+\nu\right)^{r(0)}}-\frac{1}{\nu^{r(0)}}\right] .
\end{gathered}
$$

By Lemma 8, we have

$$
\begin{aligned}
& \sum_{j=1}^{\infty}\left[\frac{r(0)}{\nu^{r(0)}} \log \left(\frac{\lambda_{j}}{\nu}+1\right)+\frac{1}{\left(\lambda_{j}+\nu\right)^{r(0)}}-\frac{1}{\nu^{r(0)}}\right] \\
& =-\nu^{-r(0)} \sum_{j=1}^{\infty}\left[-r(0) \log \left(\frac{\lambda_{j}}{\nu}+1\right)-\left(\frac{\lambda_{j}}{\nu}+1\right)^{-r(0)}+1\right] \\
& =-\nu^{-r(0)}\left(\log \operatorname{det}_{2 \mathrm{X}}\left[(\Lambda+\nu I)^{-r(0)}\right]+r(0) \log \nu\right) .
\end{aligned}
$$

Combining this with Eq. (75), we obtain

$$
\begin{aligned}
& \lim _{\beta \rightarrow 0} D_{r}^{(\alpha, \beta)}[(A+\gamma I),(B+\mu I)] \\
& =\frac{1}{\alpha^{2}}\left[\left(\nu^{-r(0)}-1\right)(1-r(0) \log \nu)-\nu^{-r(0)} \log \operatorname{det}_{2 \mathrm{X}}\left[(\Lambda+\nu I)^{-r(0)}\right]\right] .
\end{aligned}
$$

By Lemma 6, we have

$$
\begin{aligned}
\operatorname{det}_{2 \mathrm{X}}\left[(\Lambda+\nu I)^{-r(0)}\right] & =\operatorname{det}_{2 \mathrm{X}}\left(\left[(B+\mu I)^{-1}(A+\gamma I)\right]^{-r(0)}\right. \\
& =\operatorname{det}_{2 \mathrm{X}}\left(\left[(A+\gamma I)^{-1}(B+\mu I)\right]^{r(0)}\right) .
\end{aligned}
$$


Substituting this into the previous expression and $\nu=\frac{\gamma}{\mu}$, we obtain the final result.

By dual symmetry, we then obtain $\lim _{\alpha \rightarrow 0} D_{r}^{(\alpha, \beta)}[(A+\gamma I),(B+\mu I)]$ via

$$
\lim _{\alpha \rightarrow 0} D_{r}^{(\alpha, \beta)}[(A+\gamma I),(B+\mu I)]=\lim _{\alpha \rightarrow 0} D^{(\beta, \alpha)}[(B+\mu I),(A+\gamma I)] .
$$

This completes the proof.

Proof of Corollary 2. Let us prove the first statement, since the second one is entirely similar. It suffices to prove for $\alpha=1$. For $(A+\gamma I),(B+\mu I) \in \mathscr{P} \mathscr{C}_{1}(\mathcal{H})$, we have $(\Lambda+\nu I)=(B+\mu I)^{-1 / 2}(A+\gamma I)(B+\mu I)^{-1 / 2} \in \mathscr{P} \mathscr{C}_{1}(\mathcal{H})$. By Definition 3, $D_{r}^{(1,0)}[(A+\gamma I),(B+\mu I)]=\left(\nu^{-r}-1\right)(1-r \log \nu)-\nu^{-r} \log \operatorname{det}_{2 \mathrm{X}}\left[(\Lambda+\nu I)^{-r}\right]$.

By Lemma 8, we have

$$
\begin{aligned}
& \nu^{-r} \log \operatorname{det}_{2 \mathrm{X}}\left[(\Lambda+\nu I)^{-r}\right]=-r \nu^{-r} \log \operatorname{det}_{\mathrm{X}}(\Lambda+\nu I)-\nu^{-r} \operatorname{tr}\left[\left(\frac{\Lambda}{\nu}+I\right)^{-r}-I\right] \\
& =-r \nu^{-r} \log \operatorname{det}_{\mathrm{X}}(\Lambda+\nu I)-\operatorname{tr}\left[(\Lambda+\nu I)^{-r}-\nu^{-r} I\right] .
\end{aligned}
$$

It then follows that

$$
\begin{aligned}
& \left(\nu^{-r}-1\right)(1-r \log \nu)-\nu^{-r} \log \operatorname{det}_{2 \mathrm{X}}\left[(\Lambda+\nu I)^{-r}\right] \\
& =\left(\nu^{-r}-1\right)(1-r \log \nu)+r \nu^{-r} \log \operatorname{det}_{\mathrm{X}}(\Lambda+\nu I)+\operatorname{tr}\left[(\Lambda+\nu I)^{-r}-\nu^{-r} I\right] \\
& =-r\left(\nu^{-r}-1\right) \log \nu+\nu^{-r} \log \operatorname{det}_{\mathrm{X}}(\Lambda+\nu I)^{r}+\left(\nu^{-r}-1+\operatorname{tr}\left[(\Lambda+\nu I)^{-r}-\nu^{-r} I\right]\right) \\
& =-r\left(\nu^{-r}-1\right) \log \nu-\nu^{-r} \log \operatorname{det}_{\mathrm{X}}(\Lambda+\nu I)^{-r}+\operatorname{tr}_{X}\left[(\Lambda+\nu I)^{-r}-I\right] .
\end{aligned}
$$

By Lemma 8 in [2], which states that for any $\alpha \in \mathbb{R}$,

$$
\begin{gathered}
\operatorname{det}_{X}\left[(A+\gamma I)(B+\mu I)^{-1}\right]^{\alpha}=\operatorname{det}_{X}\left[(\Lambda+\nu I)^{\alpha}\right]=\operatorname{det}_{X}\left[(B+\mu I)^{-1}(A+\gamma I)\right]^{\alpha}, \\
\operatorname{tr}_{X}\left[(A+\gamma I)(B+\mu I)^{-1}\right]^{\alpha}=\operatorname{tr}_{X}\left[(\Lambda+\nu I)^{\alpha}\right]=\operatorname{tr}_{X}\left[(B+\mu I)^{-1}(A+\gamma I)\right]^{\alpha},
\end{gathered}
$$

we have

$$
\begin{gathered}
\operatorname{det}_{\mathrm{X}}(\Lambda+\nu I)^{-r}=\operatorname{det}_{\mathrm{X}}\left[(B+\mu I)^{-1}(A+\gamma I)\right]^{-r}=\operatorname{det}_{\mathrm{X}}\left[(A+\gamma I)^{-1}(B+\mu I)\right]^{r}, \\
\operatorname{tr}_{X}\left[(\Lambda+\nu I)^{-r}\right]=\operatorname{tr}_{X}\left[(B+\mu I)^{-1}(A+\gamma I)\right]^{-r}=\operatorname{tr}_{X}\left[(A+\gamma I)^{-1}(B+\mu I)\right]^{r} .
\end{gathered}
$$


Combining these with the previous expression, replacing $\nu=\frac{\gamma}{\mu}$, we obtain

$$
\begin{aligned}
D_{r}^{(1,0)}[(A+\gamma I),(B+\mu I)] & =\left(\left(\frac{\mu}{\gamma}\right)^{r}-1\right) \log \frac{\mu}{\gamma} \\
& -\left(\frac{\mu}{\gamma}\right)^{r} \log \operatorname{det}_{\mathrm{X}}\left[(A+\gamma I)^{-1}(B+\mu I)\right]^{r} \\
& +\operatorname{tr}_{X}\left(\left[(A+\gamma I)^{-1}(B+\mu I)\right]^{r}-I\right) .
\end{aligned}
$$

This completes the proof.

Proof of Theorem 3. The proof is identical to the proof in the setting $(A+\gamma I),(B+$ $\mu I) \in \mathscr{P} \mathscr{C}_{1}(\mathcal{H})$ (Theorem 9 in [2]).

Proof of Theorem 4. This follows from the dual symmetry in Theorem 6 and the limiting behavior in Theorem 3 .

\subsection{Proofs of the properties of the Log-Determinant divergences}

For the proof on Theorem 5 on positivity, we first need the following technical results.

Lemma 11. Assume that $\gamma>0, \alpha>0, \beta>0$ are fixed. Let $r \in \mathbb{R}, r \neq 0$ be fixed. Then for $\delta=\frac{\alpha \gamma^{r}}{\alpha \gamma^{r}+\beta}, \quad p=r(1-\delta), q=r \delta$, we have

$$
\frac{r\left(\delta-\frac{\alpha}{\alpha+\beta}\right)}{\alpha \beta} \log \gamma+\frac{1}{\alpha \beta} \log \left(\frac{\alpha \gamma^{p}+\beta \gamma^{-q}}{\alpha+\beta}\right) \geq 0 .
$$

Equality happens if and only if $\gamma=1$.

Proof of Lemma 11. By the strict concavity of the log function, we have

$$
\log \left(\frac{\alpha \gamma^{p}+\beta \gamma^{-q}}{\alpha+\beta}\right) \geq \frac{(p \alpha-q \beta) \log \gamma}{\alpha+\beta},
$$

with equality if and only if $\gamma^{p}=\gamma^{-q} \Longleftrightarrow \gamma^{p+q}=\gamma^{r}=1$. Since $\gamma>0$ and $r \neq 0$, this happens if and only if $\gamma=1$. Thus we have

$$
\begin{aligned}
& \frac{r\left(\delta-\frac{\alpha}{\alpha+\beta}\right)}{\alpha \beta} \log \gamma+\frac{1}{\alpha \beta} \log \left(\frac{\alpha \gamma^{p}+\beta \gamma^{-q}}{\alpha+\beta}\right) \\
& \geq \frac{1}{\alpha \beta}\left[r\left(\delta-\frac{\alpha}{\alpha+\beta}\right)+\frac{p \alpha-q \beta}{\alpha+\beta}\right] \log \gamma \\
& =\frac{1}{\alpha \beta}\left[q-\frac{(p+q) \alpha}{\alpha+\beta}+\frac{p \alpha-q \beta}{\alpha+\beta}\right] \log \gamma=0, \text { since } r=p+q .
\end{aligned}
$$

Equality happens if and only if $\gamma=1$. 
Lemma 12. Assume that $\gamma>0, \alpha>0, \beta>0$ are fixed. Let $r \in \mathbb{R}, r \neq 0$ be fixed. Assume that $\lambda \in \mathbb{R}$ is also fixed, such that $\lambda+\gamma>0$. Then for $\delta=\frac{\alpha \gamma^{r}}{\alpha \gamma^{r}+\beta}, \quad p=$ $r(1-\delta), q=r \delta$,

$$
\log \left(\frac{\alpha(\lambda+\gamma)^{p}+\beta(\lambda+\gamma)^{-q}}{\alpha \gamma^{p}+\beta \gamma^{-q}}\right) \geq 0
$$

Equality happens if and only if $\lambda=0$.

Proof of Lemma 12. By the strict concavity of the log function, we have

$$
\begin{aligned}
& \log \left(\frac{\alpha(\lambda+\gamma)^{p}+\beta(\lambda+\gamma)^{-q}}{\alpha \gamma^{p}+\beta \gamma^{-q}}\right) \\
& =\log \left[\frac{\alpha \gamma^{p}}{\alpha \gamma^{p}+\beta \gamma^{-q}}\left(\frac{\lambda}{\gamma}+1\right)^{p}+\frac{\beta \gamma^{-q}}{\alpha \gamma^{p}+\beta \gamma^{-q}}\left(\frac{\lambda}{\gamma}+1\right)^{-q}\right] \\
& \geq \frac{p \alpha \gamma^{p}-q \beta \gamma^{-q}}{\alpha \gamma^{p}+\beta \gamma^{-q}} \log \left(\frac{\lambda}{\gamma}+1\right)=0,
\end{aligned}
$$

since $p \alpha \gamma^{p}-q \beta \gamma^{-q}=0$, as can be verified directly using the given hypothesis. Equality happens if and only if $\left(\frac{\lambda}{\gamma}+1\right)^{p}=\left(\frac{\lambda}{\gamma}+1\right)^{-q} \Longleftrightarrow\left(\frac{\lambda}{\gamma}+1\right)^{p+q}=\left(\frac{\lambda}{\gamma}+1\right)^{r}=1$. Since $\lambda+\gamma>0, \gamma>0$, and $r \neq 0$, this happens if and only if $\lambda=0$.

Proof of Theorem 5 (Positivity). (a) The case $\alpha>0, \beta>0$.

Let $\Lambda+\frac{\gamma}{\mu} I=(B+\mu I)^{-1 / 2}(A+\gamma I)(B+\mu I)^{-1 / 2}$. Let $\left\{\lambda_{j}\right\}_{j=1}^{\infty}$ be the eigenvalues of $\Lambda$. By Theorem 8 in [2], we have the expansion

$$
\begin{aligned}
D_{r}^{(\alpha, \beta)}[(A+\gamma I),(B+\mu I)] & =\frac{r\left(\delta-\frac{\alpha}{\alpha+\beta}\right)}{\alpha \beta} \log \left(\frac{\gamma}{\mu}\right)+\frac{1}{\alpha \beta} \log \left(\frac{\alpha\left(\frac{\gamma}{\mu}\right)^{p}+\beta\left(\frac{\gamma}{\mu}\right)^{-q}}{\alpha+\beta}\right) \\
& +\frac{1}{\alpha \beta} \log \operatorname{det}\left(\frac{\alpha\left(\Lambda+\frac{\gamma}{\mu} I\right)^{p}+\beta\left(\Lambda+\frac{\gamma}{\mu} I\right)^{-q}}{\alpha\left(\frac{\gamma}{\mu}\right)^{p}+\beta\left(\frac{\gamma}{\mu}\right)^{-q}}\right) \\
& =\frac{r\left(\delta-\frac{\alpha}{\alpha+\beta}\right)}{\alpha \beta} \log \left(\frac{\gamma}{\mu}\right)+\frac{1}{\alpha \beta} \log \left(\frac{\alpha\left(\frac{\gamma}{\mu}\right)^{p}+\beta\left(\frac{\gamma}{\mu}\right)^{-q}}{\alpha+\beta}\right) \\
& +\frac{1}{\alpha \beta} \sum_{j=1}^{\infty} \log \left(\frac{\alpha\left(\lambda_{j}+\frac{\gamma}{\mu}\right)^{p}+\beta\left(\lambda_{j}+\frac{\gamma}{\mu}\right)^{-q}}{\alpha\left(\frac{\gamma}{\mu}\right)^{p}+\beta\left(\frac{\gamma}{\mu}\right)^{-q}}\right),
\end{aligned}
$$

where $p=p(\beta)=r(1-\delta)=\frac{r \beta}{\alpha\left(\frac{\gamma}{\mu}\right)^{r}+\beta}, q=q(\beta)=r \delta=\frac{r \alpha\left(\frac{\gamma}{\mu}\right)^{r}}{\alpha\left(\frac{\gamma}{\mu}\right)^{r}+\beta}$.

By Lemma 11, we have

$$
\frac{r\left(\delta-\frac{\alpha}{\alpha+\beta}\right)}{\alpha \beta} \log \left(\frac{\gamma}{\mu}\right)+\frac{1}{\alpha \beta} \log \left(\frac{\alpha\left(\frac{\gamma}{\mu}\right)^{p}+\beta\left(\frac{\gamma}{\mu}\right)^{-q}}{\alpha+\beta}\right) \geq 0,
$$


with equality if and only if $\frac{\gamma}{\mu}=1 \Longleftrightarrow \gamma=\mu$.

By Lemma 12, we have $\forall j \in \mathbb{N}$,

$$
\log \left(\frac{\alpha\left(\lambda_{j}+\frac{\gamma}{\mu}\right)^{p}+\beta\left(\lambda_{j}+\frac{\gamma}{\mu}\right)^{-q}}{\alpha\left(\frac{\gamma}{\mu}\right)^{p}+\beta\left(\frac{\gamma}{\mu}\right)^{-q}}\right) \geq 0,
$$

with equality if and only $\lambda_{j}=0$.

Combining these two results with the previous expression for $D_{r}^{(\alpha, \beta)}[(A+\gamma I),(B+$ $\mu I)]$, we obtain

$$
D_{r}^{(\alpha, \beta)}[(A+\gamma I),(B+\mu I)] \geq 0,
$$

with equality if and only if $\gamma=\mu$ and $\lambda_{j}=0 \forall j \in \mathbb{N}$, that is $\Lambda=0$. This is equivalent to $(B+\mu I)^{-1 / 2}(A+\gamma I)(B+\mu I)^{-1 / 2}=I \Longleftrightarrow(A+\gamma I)=(B+\mu I) \Longleftrightarrow A=$ $B, \gamma=\mu$, since $A, B \in \operatorname{HS}(\mathcal{H})$ by assumption.

(b) The case $\alpha=0, \beta>0$.

Since the factor $\beta^{2}$ can be ignored, it suffices to consider the case $\beta=1$. We have

$$
\begin{aligned}
D_{r}^{(0,1)}[(A+\gamma I),(B+\mu I)] & =\left[\left(\frac{\gamma}{\mu}\right)^{r}-1\right]\left(1+r \log \frac{\gamma}{\mu}\right) \\
& -\left(\frac{\gamma}{\mu}\right)^{r} \log \operatorname{det}_{2 \mathrm{X}}\left(\left[(B+\mu I)^{-1}(A+\gamma I)\right]^{r}\right) .
\end{aligned}
$$

By Lemma 6, we have for any $r \in \mathbb{R}$,

$\operatorname{det}_{2 \mathrm{X}}\left(\left[(B+\mu I)^{-1}(A+\gamma I)\right]^{r}=\operatorname{det}_{2 \mathrm{X}}\left[\left(\Lambda+\frac{\gamma}{\mu} I\right)^{r}\right]=\operatorname{det}_{2 \mathrm{X}}\left[\left(\frac{\gamma}{\mu}\right)^{r}\left(\frac{\mu}{\gamma} \Lambda+I\right)^{r}\right]\right.$.

By the Factorization Rule in Lemma 3, we then have

$$
\operatorname{det}_{2 \mathrm{X}}\left(\left[(B+\mu I)^{-1}(A+\gamma I)\right]^{r}=\left(\frac{\gamma}{\mu}\right)^{r} \operatorname{det}_{2}\left[\left(\frac{\mu}{\gamma} \Lambda+I\right)^{r}\right] .\right.
$$

Combining this with the first expression for $D_{r}^{(0,1)}[(A+\gamma I),(B+\mu I)]$, we obtain

$$
\begin{aligned}
D_{r}^{(0,1)}[(A+\gamma I),(B+\mu I)] & =\left(\frac{\gamma}{\mu}\right)^{r}-1-r \log \frac{\gamma}{\mu} \\
& -\left(\frac{\gamma}{\mu}\right)^{r} \log \operatorname{det}_{2}\left[\left(\frac{\mu}{\gamma} \Lambda+I\right)^{r}\right] .
\end{aligned}
$$

By Lemma 7, we have

$$
\log \operatorname{det}_{2}\left[\left(\frac{\mu}{\gamma} \Lambda+I\right)^{r}\right] \leq 0, \text { with equality if and only if } \Lambda=0 .
$$


By Lemma 9, we have

$$
\left(\frac{\gamma}{\mu}\right)^{r}-1-r \log \frac{\gamma}{\mu} \geq 0, \text { with equality if and only if } \frac{\gamma}{\mu}=1 .
$$

Together with the previous expression for $D_{r}^{(0,1)}[(A+\gamma I),(B+\mu I)]$, these imply

$$
\operatorname{det}_{2 \mathrm{X}}\left(\left[(B+\mu I)^{-1}(A+\gamma I)\right]^{r} \geq 0\right.
$$

with equality if and only if $\Lambda+\frac{\gamma}{\mu} I=(B+\mu I)^{-1 / 2}(A+\gamma I)(B+\mu I)^{-1 / 2}=I \Longleftrightarrow$ $A+\gamma I=B+\mu I \Longleftrightarrow A=B, \gamma=\mu$.

(c) The case $\alpha>0, \beta=0$ follows from the previous case by dual symmetry. This completes the proof.

Proof of Theorem 6 (Dual symmetry). For the case $\alpha>0, \beta>0$, the proof is identical to that for the setting $(A+\gamma I),(B+\mu I) \in \mathscr{P} \mathscr{C}_{1}(\mathcal{H})$ (Theorem 13 in [2]). The cases $\alpha=0, \beta>0$ and $\alpha>0, \beta=0$ are obvious from Eqs. (60) and (61).

Proof of Theorem 7 (Dual invariance under inversion). For the case $\alpha>0, \beta>0$, the proof is identical to that for the setting $(A+\gamma I),(B+\mu I) \in \mathscr{P} \mathscr{C}_{1}(\mathcal{H})$ (Theorem 14 in [2]).

Consider the case $\alpha=0, \beta>0$ (the case $\alpha>0, \beta=0$ follows from dual symmetry). It suffices to consider $\beta=1$. We have

$$
(A+\gamma I)^{-1}=\frac{1}{\gamma} I-\frac{A}{\gamma}(A+\gamma I)^{-1}, \quad(B+\mu I)^{-1}=\frac{1}{\mu} I-\frac{B}{\mu}(B+\mu I)^{-1} .
$$

By Eq. (61), we have

$$
\begin{aligned}
& D_{r}^{(0,1)}\left[(A+\gamma I)^{-1},(B+\mu I)^{-1}\right]=\left[\left(\frac{1 / \gamma}{1 / \mu}\right)^{r}-1\right]\left(1-r \log \frac{1 / \gamma}{1 / \mu}\right) \\
& -\left(\frac{1 / \gamma}{1 / \mu}\right)^{r} \log \operatorname{det}_{2 \mathrm{X}}\left(\left[(B+\mu I)(A+\gamma I)^{-1}\right]^{r}\right) \\
& =\left[\left(\frac{\mu}{\gamma}\right)^{r}-1\right]\left(1-r \log \frac{\mu}{\gamma}\right)-\left(\frac{\mu}{\gamma}\right)^{r} \log \operatorname{det}_{2 \mathrm{X}}\left(\left[(A+\gamma I)(B+\mu I)^{-1}\right]^{-r}\right) \\
& =\left[\left(\frac{\gamma}{\mu}\right)^{-r}-1\right]\left(1+r \log \frac{\gamma}{\mu}\right)-\left(\frac{\gamma}{\mu}\right)^{-r} \log \operatorname{det}_{2 \mathrm{X}}\left(\left[(B+\mu I)^{-1}(A+\gamma I)\right]^{-r}\right) \\
& =D_{-r}^{(0,1)}[(A+\gamma I),(B+\mu I)],
\end{aligned}
$$


where we have used the property $\operatorname{det}_{2 \mathrm{X}}\left(\left[(A+\gamma I)(B+\mu I)^{-1}\right]^{-r}\right)=\operatorname{det}_{2 \mathrm{X}}([(B+$ $\left.\left.\mu I)^{-1}(A+\gamma I)\right]^{-r}\right)$ by Lemma 6 . This completes the proof.

Proof of Theorem 8 (Affine invariance). For any $(A+\gamma I),(B+\mu I) \in \mathscr{P} \mathscr{C}_{2}(\mathcal{H})$, and any $(C+\nu I) \in \operatorname{HS}_{X}(\mathcal{H})$, we have

$$
\begin{aligned}
& (C+\nu I)(A+\gamma I)(C+\nu I)^{*} \\
& =C A C^{*}+\nu\left(C A+A C^{*}\right)+\nu^{2} A+\gamma C C^{*}+\gamma \nu\left(C+C^{*}\right)+\gamma \nu^{2} I \in \mathscr{P} \mathscr{C}_{2}(\mathcal{H}), \\
& (C+\nu I)(B+\mu I)(C+\nu I)^{*} \\
& =C B C^{*}+\nu\left(C B+B C^{*}\right)+\nu^{2} B+\mu C C^{*}+\mu \nu\left(C+C^{*}\right)+\mu \nu^{2} I \in \mathscr{P} \mathscr{C}_{2}(\mathcal{H}),
\end{aligned}
$$

For two operators $(A+\gamma I),(B+\mu I) \in \mathscr{P} \mathscr{C}_{2}(\mathcal{H})$, we then have

$$
\begin{aligned}
& {\left[(C+\nu I)(A+\gamma I)(C+\nu I)^{*}\right]\left[(C+\nu I)(B+\mu I)(C+\nu I)^{*}\right]^{-1}} \\
& =(C+\nu I)\left[(A+\gamma I)(B+\mu I)^{-1}\right](C+\nu I)^{-1} .
\end{aligned}
$$

Then for any $p \in \mathbb{R}$, we have by Proposition 1 ,

$$
\begin{aligned}
& \left(\left[(C+\nu I)(A+\gamma I)(C+\nu I)^{*}\right]\left[(C+\nu I)(B+\mu I)(C+\nu I)^{*}\right]^{-1}\right)^{p} \\
& =\left[(C+\nu I)\left[(A+\gamma I)(B+\mu I)^{-1}\right](C+\nu I)^{-1}\right]^{p} \\
& =(C+\nu I)\left[(A+\gamma I)(B+\mu I)^{-1}\right]^{p}(C+\nu I)^{-1} \in \operatorname{HS}_{X}(\mathcal{H})
\end{aligned}
$$

Thus for the cases $\alpha=0, \beta>0$ and $\alpha>0, \beta=0$, the affine-invariance follows from the Similarity Invariance of the extended Hilbert-Carleman determinant $\operatorname{det}_{2 \mathrm{X}}$, stated in Lemma 4, along with the invariance of the ratio $\frac{\gamma \nu^{2}}{\mu \nu^{2}}=\frac{\gamma}{\nu}$.

For the case $\alpha>0, \beta>0$, let $a=\frac{\alpha}{\alpha+\beta}, b=\frac{\beta}{\alpha+\beta}, p=r(1-\delta), q=r \delta$, we have by Proposition 3

$$
a\left[(A+\gamma I)(B+\mu I)^{-1}\right]^{p}+b\left[(A+\gamma I)(B+\mu I)^{-1}\right]^{-q} \in \operatorname{Tr}_{X}(\mathcal{H}) .
$$

It follows then that

$$
\begin{aligned}
& a\left(\left[(C+\nu I)(A+\gamma I)(C+\nu I)^{*}\right]\left[(C+\nu I)(B+\mu I)(C+\nu I)^{*}\right]^{-1}\right)^{p} \\
& +b\left(\left[(C+\nu I)(A+\gamma I)(C+\nu I)^{*}\right]\left[(C+\nu I)(B+\mu I)(C+\nu I)^{*}\right]^{-1}\right)^{-q} \\
& =(C+\nu I)\left(a\left[(A+\gamma I)(B+\mu I)^{-1}\right]^{p}+b\left[(A+\gamma I)(B+\mu I)^{-1}\right]^{-q}\right)(C+\nu I)^{-1} \\
& \in \operatorname{Tr}_{X}(\mathcal{H}) .
\end{aligned}
$$


From the Similarity Invariance of both the extended Fredholm determinant $\operatorname{det}_{\mathrm{X}}$, stated in Eq. (34), along with the invariance of the ratio $\frac{\gamma \nu^{2}}{\mu \nu^{2}}=\frac{\gamma}{\nu}$, we obtain the affineinvariance for $D_{r}^{(\alpha, \beta)}[(A+\gamma I),(B+\mu I)]$.

Proof of Theorem 9 (Unitary invariance). The proof for this theorem is similar to that of Theorem 9, by utilizing the fact that $C^{*}=C^{-1}$ and the Similarity Invariance

$$
\operatorname{det}_{\mathrm{X}}\left[C(A+\gamma I) C^{-1}\right]=\operatorname{det}_{\mathrm{X}}(A+\gamma I), \quad A+\gamma I \in \operatorname{Tr}_{X}(\mathcal{H})
$$

for the case $\alpha>0, \beta>0$, and

$$
\operatorname{det}_{2 \mathrm{X}}\left[C(A+\gamma I) C^{-1}\right]=\operatorname{det}_{2 \mathrm{X}}(A+\gamma I), \quad A+\gamma I \in \mathrm{HS}_{X}(\mathcal{H}),
$$

for the cases $\alpha>0, \beta=0$ and $\alpha=0, \beta>0$.

\section{References}

[1] H. Minh, Infinite-dimensional Log-Determinant divergences between positive definite trace class operators, Linear Algebra and Its Applications (In Press) (2016) http://dx.doi.org/10.1016/j.laa.2016.09.018.

[2] H. Minh, Infinite-dimensional Log-Determinant divergences II: Alpha-Beta divergences, arXiv preprint arXiv:1610.08087v2.

[3] A. Cichocki, S. Cruces, S. Amari, Log-Determinant divergences revisited: AlphaBeta and Gamma Log-Det divergences, Entropy 17 (5) (2015) 2988-3034.

[4] R. Bhatia, Positive Definite Matrices, Princeton University Press, 2007.

[5] G. Mostow, Some new decomposition theorems for semi-simple groups, Memoirs of the American Mathematical Society 14 (1955) 31-54.

[6] J. D. Lawson, Y. Lim, The geometric mean, matrices, metrics, and more, The American Mathematical Monthly 108 (9) (2001) 797-812.

[7] X. Pennec, P. Fillard, N. Ayache, A Riemannian framework for tensor computing, International Journal of Computer Vision 66 (1) (2006) 41-66. 
[8] D. A. Bini, B. Iannazzo, Computing the Karcher mean of symmetric positive definite matrices, Linear Algebra and its Applications 438 (4) (2013) 1700-1710.

[9] Z. Chebbi, M. Moakher, Means of Hermitian positive-definite matrices based on the log-determinant $\alpha$-divergence function, Linear Algebra and its Applications 436 (7) (2012) 1872-1889.

[10] S. Sra, A new metric on the manifold of kernel matrices with application to matrix geometric means, in: Advances in Neural Information Processing Systems (NIPS), 2012, pp. 144-152.

[11] G. Larotonda, Nonpositive curvature: A geometrical approach to Hilbert-Schmidt operators, Differential Geometry and its Applications 25 (2007) 679-700.

[12] N. Dunford, J. Schwartz, Linear Operators, Part 2: Spectral Theory, Self Adjoint Operators in Hilbert Space, Wiley, 1988.

[13] B. Simon, Notes on infinite determinants of Hilbert space operators, Advances in Mathematics 24 (1977) 244-273.

[14] K. Fan, On a theorem of Weyl concerning eigenvalues of linear transformations: II, Proceedings of the National Academy of Sciences of the United States of America 36 (1) (1950) 31.

[15] H. Q. Minh, V. Murino, From covariance matrices to covariance operators: Data representation from finite to infinite-dimensional settings, in: H. Q. Minh, V. Murino (Eds.), Algorithmic Advances in Riemannian Geometry and Applications: For Machine Learning, Computer Vision, Statistics, and Optimization, Springer International Publishing, Cham, 2016, pp. 115-143. 